\title{
TREM2 sustains macrophage-hepatocyte metabolic coordination in nonalcoholic fatty liver disease and sepsis
}

\author{
Jinchao Hou, ${ }^{1,2}$ Jue Zhang, ${ }^{1}$ Ping Cui, ${ }^{1,3}$ Yingyue Zhou, ${ }^{2}$ Can Liu, ${ }^{4,5}$ Xiaoliang Wu, ${ }^{6}$ Yun Ji, ${ }^{7}$ Sicong Wang, ${ }^{1}$ Baoli Cheng, ${ }^{1}$ Hui Ye, ${ }^{1}$ \\ Liqi Shu, ${ }^{8}$ Kai Zhang, ${ }^{1}$ Di Wang, ${ }^{9}$ Jielin Xu, ${ }^{10}$ Qiang Shu, ${ }^{4}$ Marco Colonna, ${ }^{2}$ and Xiangming Fang ${ }^{1}$ \\ 'Department of Anesthesiology, The First Affiliated Hospital, Zhejiang University School of Medicine, Hangzhou, Zhejiang, China. ${ }^{2}$ Department of Pathology and Immunology, Washington University \\ School of Medicine, St. Louis, Missouri, USA. ${ }^{3}$ Translational Research Program, Department of Anesthesiology \& Center for Shock Trauma and Anesthesiology Research, University of Maryland School \\ of Medicine, Baltimore, Maryland, USA. ${ }^{4}$ Department of Thoracic and Cardiovascular Surgery, The Children's Hospital, Zhejiang University School of Medicine, Hangzhou, Zhejiang, China. ${ }^{5}$ Department \\ of Anesthesiology, Yijishan Hospital of Wannan Medical College, Wuhu, Anhui, China. ${ }^{6}$ Department of Intensive Care Medicine, The First Affiliated Hospital, Zhejiang University School of Medicine, \\ Hangzhou, Zhejiang, China. ${ }^{7}$ Surgical Intensive Care Unit, The Second Affiliated Hospital, Zhejiang University School of Medicine, Hangzhou, Zhejiang, China. ${ }^{8}$ Department of Neurology, The Warren \\ Alpert Medical School of Brown University, Providence, Rhode Island, USA. Institute of Immunology, Zhejiang University School of Medicine, Hangzhou, Zhejiang, China. ${ }^{10} \mathrm{C}$ enomic Medicine Institute, \\ Lerner Research Institute, Cleveland Clinic, Cleveland, Ohio, USA.
}

\begin{abstract}
Sepsis is a leading cause of death in critical illness, and its pathophysiology varies depending on preexisting medical conditions. Here we identified nonalcoholic fatty liver disease (NAFLD) as an independent risk factor for sepsis in a large clinical cohort and showed a link between mortality in NAFLD-associated sepsis and hepatic mitochondrial and energetic metabolism dysfunction. Using in vivo and in vitro models of liver lipid overload, we discovered a metabolic coordination between hepatocyte mitochondria and liver macrophages that express triggering receptor expressed on myeloid cells-2 (TREM2). Trem2-deficient macrophages released exosomes that impaired hepatocytic mitochondrial structure and energy supply because of their high content of miR-106b-5p, which blocks Mitofusin 2 (Mfn2). In a mouse model of NAFLDassociated sepsis, TREM2 deficiency accelerated the initial progression of NAFLD and subsequent susceptibility to sepsis. Conversely, overexpression of TREM2 in liver macrophages improved hepatic energy supply and sepsis outcome. This study demonstrates that NAFLD is a risk factor for sepsis, providing a basis for precision treatment, and identifies hepatocytemacrophage metabolic coordination and TREM2 as potential targets for future clinical trials.
\end{abstract}

\section{Introduction}

Sepsis is a lethal syndrome characterized by infection and organ dysfunction, affecting approximately 19 million individuals per year worldwide (1). The heterogeneity of septic patients makes clinical characteristics and disease process extremely variable $(2,3)$. Awareness of the precise characteristics and pathogenesis is essential for effective antisepsis treatment. In this context, improving definition of patient phenotype is needed to allow for individualized management strategies matched to a patient's molecular and biochemical profile. Nonalcoholic fatty liver disease (NAFLD) is the most common chronic liver disease in the world, affecting up to $30 \%$ of the adult population $(4,5)$. NAFLD encompasses a histological spectrum, ranging from simple steatosis to nonalcoholic steatohepatitis (NASH), the latter of which is characterized by advanced hepatic inflammation, endoplasmic

Authorship note: $\mathrm{JH}, \mathrm{JZ}$, and PC contributed equally to this work.

Conflict of interest: $\mathrm{MC}$ received research support from Alector, Amgen, Ono, and

Pfizer. He is a scientific advisory board member for Vigil, Cell Signaling Technologies, and NGMBio, and has a patent pending for TREM2 ("Compositions and methods for reducing resistance to or enhancing immunotherapy," no. 62/981,827).

Copyright: ( 2021, American Society for Clinical Investigation.

Submitted: November 22, 2019; Accepted: December 16, 2020; Published: February 15, 2021.

Reference information: J Clin Invest. 2021;131(4):e135197.

https://doi.org/10.1172/JCI135197. reticulum (ER) stress, mitochondria malfunction, and fibrosis (6). The established 2-hit model postulates that liver fat buildup makes hepatocytes vulnerable to second injury (7). Accordingly, NAFLD is associated with adverse outcomes in many diseases (8-11).

In this study, we initially demonstrated a role of NAFLD in septic mortality in a large clinical cohort, supporting that compromised hepatic homeostasis is an important component of the maladaptive host response to sepsis. By investigating the underlying mechanisms, we found that NAFLD is associated with impaired hepatic mitochondrial homeostasis and energetic metabolism, as well as the emergence of liver macrophages expressing triggering receptor expressed on myeloid cells-2 (TREM2). TREM2 is a lipid-binding cell surface receptor that transmits intracellular signals through the adapter DAP12. TREM2 has been previously shown to sustain metabolic fitness of microglia during Alzheimer's disease and to support the ability of adipose tissue macrophages to control metabolic syndrome in high fat diet (HFD) (12-15). Using in vitro and in vivo models, we found that liver control of lipid overload involves a metabolic coordination between macrophages and hepatocytes that depends in part on TREM2. Trem2-deficient macrophages released exosomes (Exos) that impaired the mitochondria and energy supply of hepatocytes because of their high content of miR-106b-5p, which blocks Mfn2. TREM2 deficiency accelerated the progression of NAFLD and aggravated NAFLD-associated sepsis in vivo, whereas TREM2 overexpression conversely had a protective effect in these 
models. These results demonstrate that NAFLD is a risk factor for sepsis, providing a basis for precision treatment, and identify TREM2-dependent hepatocyte-macrophage metabolic coordination as a mechanism that can be targeted to improve NAFLD and NAFLD-associated pathology, such as sepsis.

\section{Results}

NAFLD is an independent risk factor for hospital mortality in critically ill patients with sepsis. To investigate the contribution of NAFLD to the mortality of sepsis, we performed a prospective clinical cohort study. A total of 1307 patients diagnosed with sepsis according to Sepsis 3.0 criteria were encountered, of which 524 (40.09\%) patients with sepsis who had computed tomography (CT) of the abdomen within 2 months prior to ICU admission or during ICU stay were included. The flow diagram of patient enrollment and exclusion is presented in Figure 1A. The CT diagnosis of hepatic steatosis was made by measuring liver attenuation (LA) in Hounsfield units (HU). NAFLD was diagnosed when the LA value was $\leq 40$ $\mathrm{HU}$ (hepatic steatosis area $>30 \%$, moderate-to-severe, Figure 1B, and refs. 16-19). Of these 524 septic patients, 129 (24.62\%) were identified as NAFLD. The clinical characteristics of 524 adults with sepsis according to hepatic steatosis categories are summarized in Supplemental Table 1; supplemental material available online with this article; https://doi.org/10.1172/JCI135197DS1.

The primary outcomes were 28-day mortality and hospital mortality after admission to the ICU. Secondary outcomes were the differences in the need for ventilation, renal replacement therapy (RRT), vasopressor infusions, immunomodulator drugs, and ICU care according to the hepatic steatosis categories at an interim 28-day time point. Overall, the primary unfavorable outcome occurred in 104 (28-day mortality, 19.85\%) and 121 (hospital mortality, $23.09 \%$ ) of 524 patients with sepsis.

The NAFLD cohort showed significantly increased 28-day mortality and hospital mortality (32.56\% vs. $15.7 \%, P<0.0001 ; 37.21 \%$ vs. $18.48 \%, P<0.0001$, respectively) compared with the mortality of the non-NAFLD cohort (Figure 1, C and D). Multivariate logistic regression was further performed to estimate the effect of NAFLD on hospital mortality adjusted by age, sex, BMI, and site of infection, and it showed that NAFLD was an independent risk factor (Figure 1E). The adjusted OR of hospital mortality was 2.92 for the NAFLD group (95\% confidence interval: 1.69-5.03, $P<0.001$ ) (Figure $1 \mathrm{E}$ ). Interestingly, the 28-day mortality and the hospital mortality of septic patients with NAFLD increased both in patients with low BMI (28-day mortality: $17.95 \%$ vs. $44.12 \%, P<0.0001$; hospital mortality: $20.77 \%$ vs. $51.47 \%, P<0.0001$ ) and those with high BMI (28-day mortality: $9.91 \%$ vs. $19.67 \%, P=0.0999$; hospital mortality: $12.61 \%$ vs. $21.31 \%, P=0.1877$, Supplemental Figure 1, A and B). Of note, in the low BMI group, the mortality rate of patients with NAFLD was approximately 2- to 2.5-fold that of patients without NAFLD (Supplemental Figure 1, A and B). Moreover, in the NAFLD group, female patients with NAFLD and patients with NAFLD who were 65 years and older showed higher 28-day and hospital mortality than male patients with NAFLD (28-day mortality: $38.33 \%$ vs. $27.54 \%, P=0.26$; hospital mortality: $46.67 \%$ vs. $28.99 \%, P=0.046)$ and patients with NAFLD who were under age 65 (28-day mortality: $50.0 \%$ vs. $19.18 \%, P=0.0003$; hospital mortality: $50.0 \%$ vs. $27.40 \%, P=0.01$ ), respectively (Supplemental Figure $1, \mathrm{C}-\mathrm{F}$ ).
Notably, the Type 2 diabetes comorbidity rate (27.91\% vs. $8.1 \%$, $P<0.001)$ and plasma glucose value $(9.72 \pm 4.14$ vs. $8.4 \pm 3.36$, $P=0.0003)$ were higher in the NAFLD group compared with the non-NAFLD group (Supplemental Table 1). Type 2 diabetes has been demonstrated to increase sepsis mortality risk (20). We repeated the analysis after excluding these diabetic patients (456 remained). The 28 -day mortality (30.11\% vs. $15.15 \%, P=0.0015)$ and hospital mortality (35.48\% vs. $17.36 \%, P=0.0003)$ were still significantly higher in the NAFLD group than in the non-NAFLD group (Supplemental Figure 1G). The same trend was observed in the diabetic patients $(n=68)$. The diabetes with NAFLD group had a higher tendency of 28-day mortality than did the diabetes without NAFLD group (28-day mortality: $38.89 \%$ vs. $21.88 \%, P=0.19$; hospital mortality: $41.67 \%$ vs. $31.25 \%, P=0.45$, Supplemental Figure $1 H$ ). In the NAFLD group, the 28-day and hospital mortality in patients with and without diabetes were comparable (28-day mortality: $38.89 \%$ vs. $30.11 \%, P=$ 0.40 ; hospital mortality: $41.67 \%$ vs. $35.48 \%, P=0.55)$, indicating a strong link between NAFLD and septic mortality.

Patients with NAFLD had higher SOFA $(8.39 \pm 4.49$ vs. 6.13 $\pm 3.53, P<0.001)$ and APACHE II (15.94 \pm 6.49 vs. $14.28 \pm 5.26$, $P=0.004)$ scores, and an increased need for immunomodulator drugs $(P<0.001)$, ventilation $(P<0.001)$, renal replace treatment $(P<0.001)$, and ICU days $(P<0.01)$ compared with patients without NAFLD (Supplemental Table 1). Alanine aminotransferase (ALT) and aspartate aminotransferase (AST) levels in patients with NAFLD were numerically increased (ALT: $185.75 \pm 638.95$ vs. $103.19 \pm 386.38, P=0.08$; and AST: $167.78 \pm 756.41$ vs. $145.04 \pm$ $456.3, P=0.07$, respectively), although these differences were not statistically significant (Supplemental Table 1). Together, our results support that NAFLD is an independent risk factor for septic death.

Patients with NAFLD exhibit liver mitochondria dysfunction. We next explored the pathology underpinning mortality risk in septic patients with NAFLD. It has been shown that liver energetic metabolism shifts from glycolysis to long-chain fatty acid oxidation (FAO) during sepsis to preserve glucose stores for the brain and provide ATP for the peripheral tissue (21-23). Mitochondria are the primary site for FAO (24). Maladaptation of hepatic mitochondria has been shown to be a central feature of the transition from simple steatosis to NASH $(25,26)$. To investigate whether the defects of energy metabolism and/or mitochondria occurs in NAFLD, we first performed transcriptome analysis from livers of donors who underwent organ donation after cardiac death (DCD; 6 non-NAFLD donors, 5 NAFLD donors). In this screen, 462 transcripts were upregulated and 447 transcripts were downregulated in response to NAFLD (Figure 2A and Supplemental Data Set 1; GSE160022). Among these differentially expressed genes (DEGs) (Padj<0.05), gene sets that contribute to endoplasmic reticulum stress, intrinsic apoptosis, and cytokine production were enriched in NAFLD livers. Conversely, gene sets that contribute to lipid metabolism were suppressed in NAFLD livers, accompanied by deficient expression of the mitochondrial matrix enzymes (Figure 2, B and C).

Next, we assessed mitochondria damage by examining hepatic mitochondrial morphology and integrity in an independent NAFLD cohort ( $n=7$ in non-NAFLD group, $n=14$ in NAFLD group). Hepatocytes in NAFLD samples displayed altered mitochondrial morphology characterized by reduced size and excessive mitochondrial damage compared with non-NAFLD controls, as shown by transmission 
A

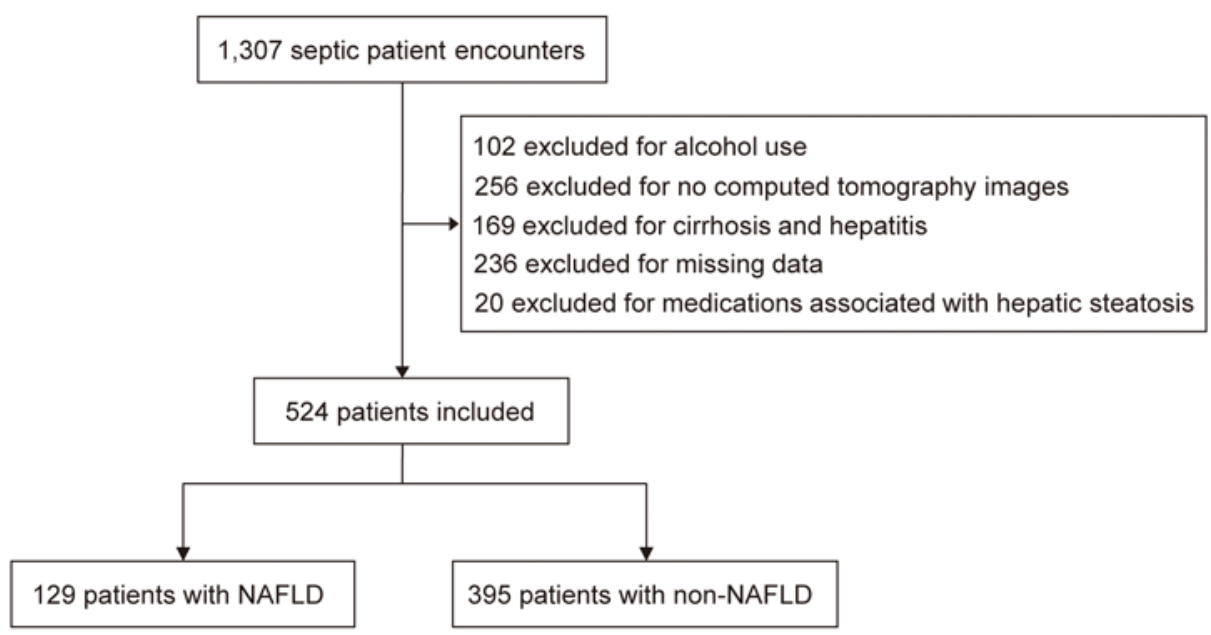

B

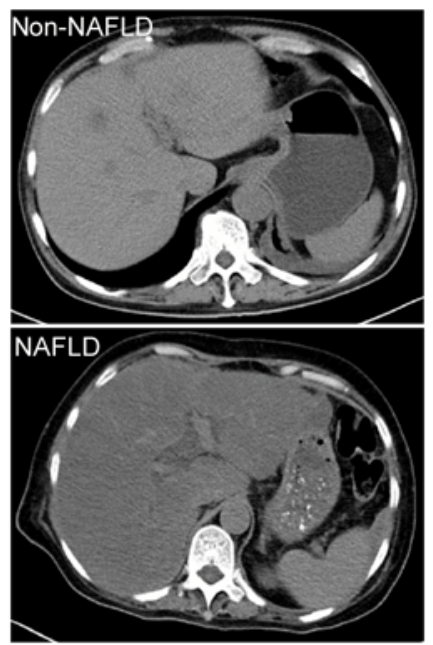

C

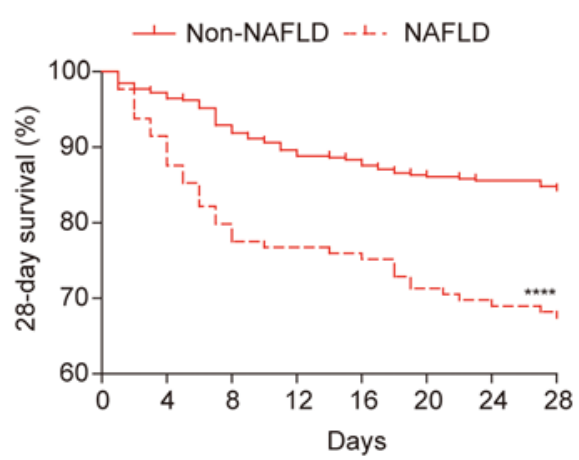

D

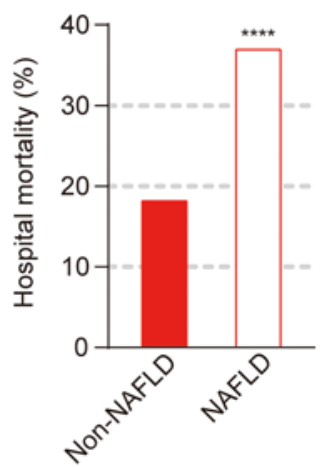

E

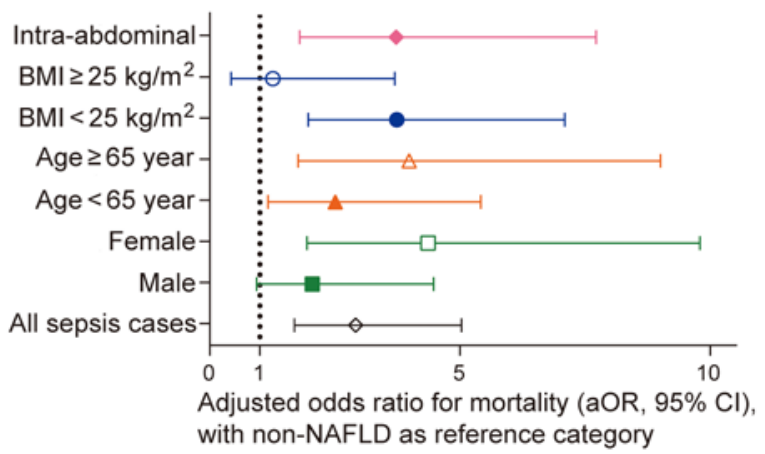

Figure 1. NAFLD is an independent risk factor for hospital mortality in critically ill patients with sepsis. (A) Study flow diagram for identification of septic patients with or without NAFLD. $n=395$ in non-NAFLD group, $n=129$ in NAFLD group. (B) Representative CT scan illustrating measurement of patients with NAFLD or not. (C and D) Kaplan-Meier survival curves (28-day) (C) and hospital mortality (D) across NAFLD category. Significant difference compared with the non-NAFLD control was determined. $n=395$ in non-NAFLD group, $n=129$ in NAFLD group. (E) Adjusted hospital mortality according to age, sex, $\mathrm{BMI}$, and site of infection. The values shown are the adjusted odds ratio (aOR, 95\% confidence interval). All sepsis cases: aOR $=2.918$ (1.693-5.031, $P<$ 0.001); male: aOR = 2.048 (0.937-4.476, $P=0.072)$; female: aOR = 4.366 (1.945-9.798, $P<0.001)$; age <65: aOR = 2.510 (1.163-5.414, $P=0.019)$; Age $\geq 65$ : aOR $=3.988$ (1.766-9.006, $P=0.001)$; BMI <25: aOR = 3.738 (1.969-7.093, $P<0.001)$; BMI $\geq 25:$ aOR = 1.261 (0.431-3.696, $P=0.672)$; intra-abdominal: aOR = 3.725 $(1.797-7.721, P<0.001)$. The data were analyzed by log-rank test $(\mathbf{C})$, or Fisher's exact test $(\mathbf{D})$, or multivariate logistic regression $(\mathbf{E}){ }^{* * * *} P<0.0001$.

electron microscopy (TEM) (Figure 2, D and E). Consistently, the NAFLD group $(n=21)$ exhibited reduced ATP content in the liver and increased ALT and AST levels in the serum compared with the non-NAFLD group ( $n=9$, Figure $2 \mathrm{~F}$ and Supplemental Table 2$)$. Therefore, our data support that increased mortality risk in septic patients with NAFLD is associated with hepatic mitochondrial dysfunction, which impairs FAO and energy supply during septic insult.

Livers of patients with NAFLD and a mouse NAFLD model have abundant TREM2 $2^{+}$macrophages. Tissue-resident macrophages are the first responding immune cells to organ injury $(27,28)$. Livers in human NASH and a mouse model of NASH exhibit a unique subset of macrophages that express triggering receptor expressed in myeloid cells 2 (TREM2) (29), a lipid receptor that controls metabolism, survival, and proliferation $(12,30)$. Whether these macrophages are beneficial or detrimental is unknown. Thus, we sought to examine the livers of patients with or without hepatic steatosis for changes in macrophage populations. Immunostaining and quantitative polymerase chain reaction (qPCR) analyses showed that the number of $\mathrm{CD}_{68}{ }^{+} \mathrm{TREM}^{+}$macrophages and the overall TREM2 expression in the NAFLD donors were significantly increased compared with controls (Figure 3, A and B). The same phenomenon was also observed in the livers of WT mice fed a HFD (Supplemental Figure 2A). Next, the monocyte-derived macrophages (MDMs) and Kupffer cells (KCs) were isolated from WT mice fed HFD for 8 weeks, and qPCR confirmed the high expression of Trem 2 in KCs (Supplemental Figure 2, B and C). It was recently reported that $\mathrm{TREM}^{+}$macrophages in the adipose tissue protect against adipocyte hypertrophy caused by HFD (13). Thus, accumulation of $\mathrm{TREM}^{+}$macrophages in NAFLD may provide a defense mechanism that attenuates liver dysmetabolism occurring in NAFLD.

TREM2 deficiency exacerbates liver mitochondria dysfunction in a NAFLD mouse model. To investigate whether TREM2 has a protective role against metabolic dysfunction in a mouse model of NAFLD, 8-week-old Trem2-deficient (Trem2--) and littermate WT mice were fed a normal chow diet (NCD) or a HFD for 8 weeks and their livers were excised and evaluated (Figure 4A). Upon 
A



$\log _{2}$ fold change (NAFLD vs. non-NAFLD)
B

Downregulation in NAFLD group

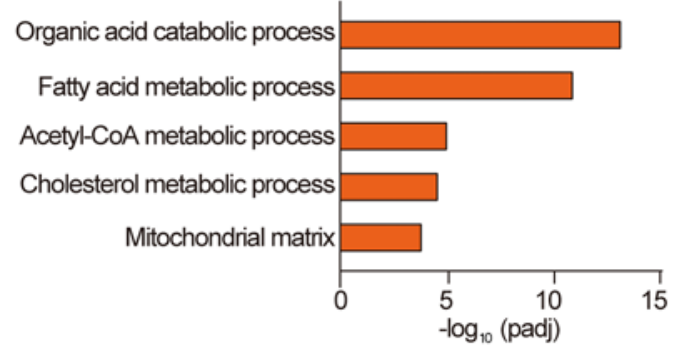

Upregulation in NAFLD group

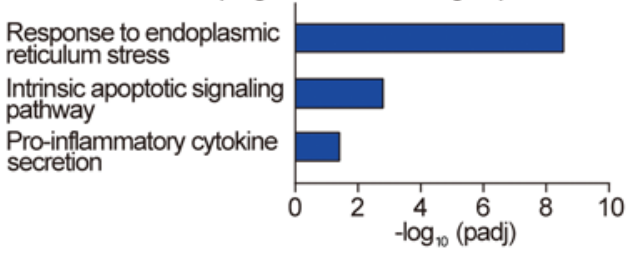

D $H \& E$ ORO TEM TEM



E

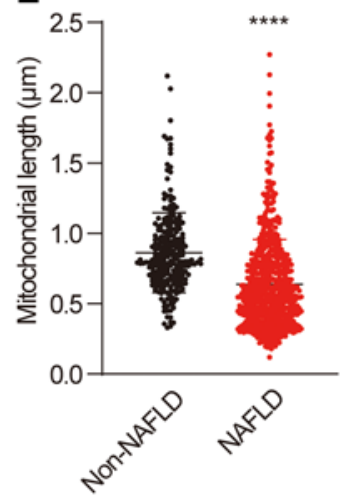

F
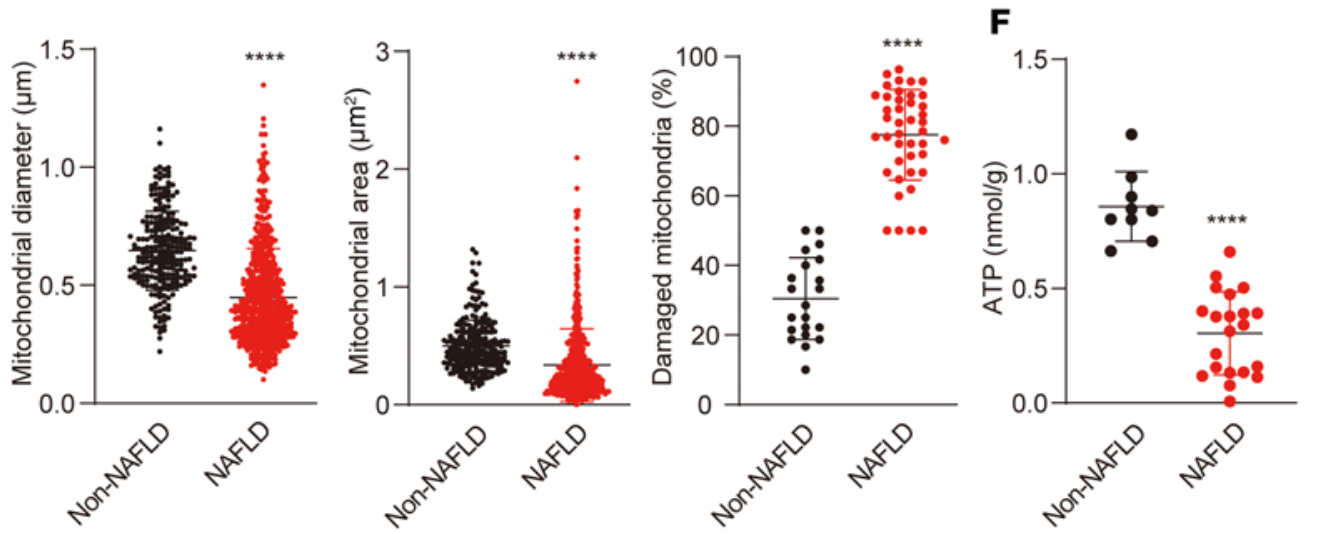

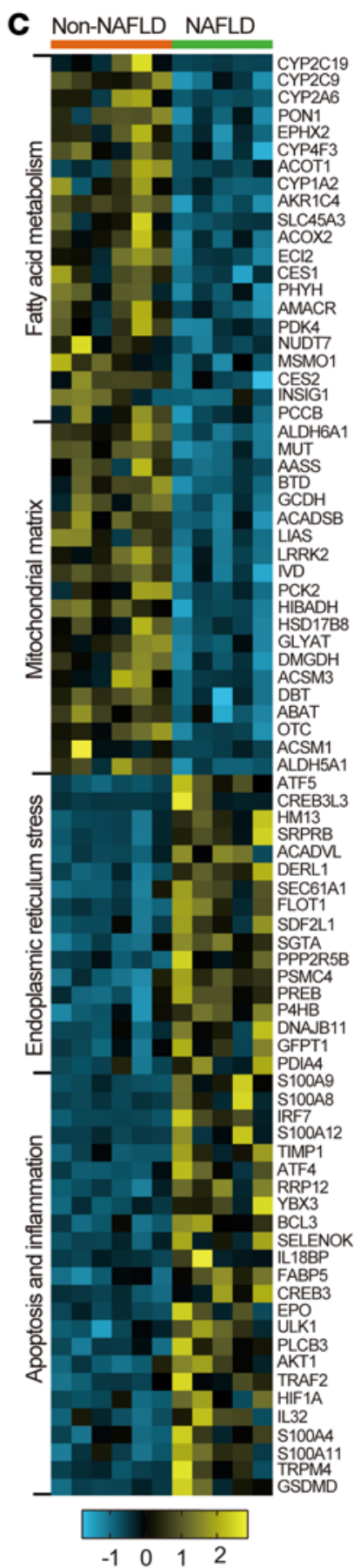

Figure 2. NAFLD patients exhibit liver mitochondria dysfunction. (A) Volcano plot showing significant DEGs (red, downregulated genes; blue, upregulated genes, Padj < 0.05) in NAFLD versus non-NAFLD livers. $n=6$ in non-NAFLD group; $n=5$ in NAFLD group. (B) GO analysis of the identified DEGs between non-NAFLD and NAFLD livers. Orange (top panel) and blue (bottom panel) indicate downregulated and upregulated GO biological processes or cellular component in NAFLD livers, respectively. (C) Heatmap showing the expression pattern of the identified DEGs that were found to be involved in fatty acid metabolism, mitochondrial matrix, endoplasmic reticulum stress, apoptosis, and inflammation. The color key indicates the expression levels. (D) Representative images of H\&E-stained and ORO-stained liver sections and TEM images from non-NAFLD and NAFLD livers. H\&E-stained and ORO-stained liver sections: $n=9$ in non-NAFLD group; $n=21$ in NAFLD group. TEM images: $n=7$ in non-NAFLD group; $n=14$ in NAFLD group. Solid and dashed white arrows refer to complete and fragmented mitochondria, respectively. Scale bars: $50 \mu \mathrm{m}, 10 \mu \mathrm{m}$, and $2 \mu \mathrm{m}$. (E) Quantification of mitochondrial parameters from liver electron microscopy images from NAFLD and non-NAFLD patients. $n=601$ mitochondria from 14 NAFLD patients or 238 mitochondria from 7 controls (3 fields/patient). (F) ATP levels in livers from non-NAFLD and NAFLD patients. $n=9$ for non-NAFLD patients; $n=21$ for NAFLD patients. All data are shown as the mean \pm SD. Significance was determined by an unpaired, 2-tailed Student's $t$ test. ${ }^{* * *} P<0.0001$. 
A
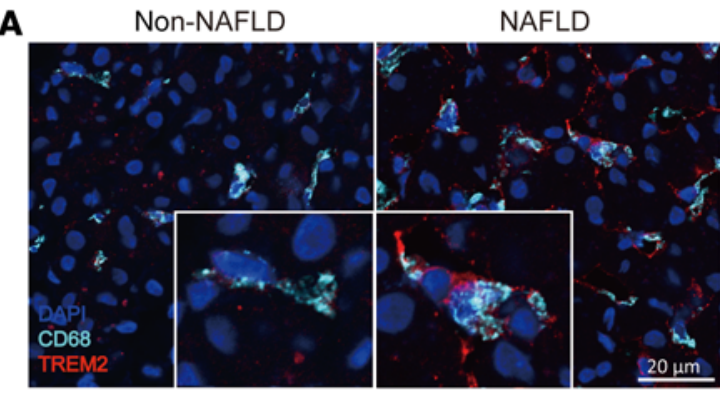

B

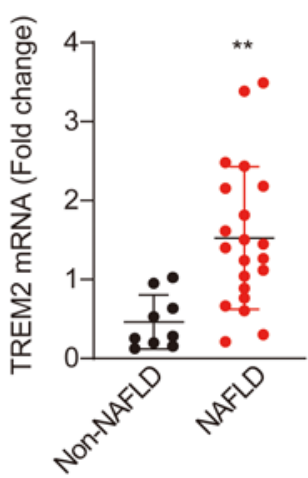

Figure 3. TREM2+ macrophages are characteristic of livers from NAFLD patients. (A) Confocal images of liver sections from non-NAFLD and NAFLD donors show CD68 (cyan), TREM2 (red) and DAPI staining. Individual CD68 ${ }^{+} \mathrm{TREM}^{+}$macrophages are depicted at a higher magnification in the inserts. Confocal images at $\times 40$ were taken with a Nikon $A 1$ inverted fluorescent microscope. $n=6$ in non-NAFLD group; $n=7$ in NAFLD group. Scale bar: $20 \mu \mathrm{m}$. (B) qPCR analysis for liver TREM2 expression normalized to $\beta$-actin mRNA levels. $n=9$ in non-NAFLD group; $n=21$ in NAFLD group. All data are shown as the mean \pm SD. Significance was determined by an unpaired, 2-tailed Student's $t$ test. ${ }^{* *} P<0.01$.

HFD feeding, Trem $2^{-/}$mice gained more body weight, epididymal fat pad weight, liver weight, and liver weight-to-body weight ratios (Figure 4, B and C; Supplemental Figure 3, A and B). Trem2 deletion increased serum and liver triglyceride (TG) by $30 \%$, as well as serum and liver cholesterol by $20 \%$ and $15 \%$, respectively (Figure 4, D and E). H\&E and Oil Red O (ORO) staining showed that Trem $2^{-/}$livers exhibited increased macrovesicular fat content, aggravated lipid droplet (LD) accumulation, and ballooning degeneration (Figure $4 \mathrm{~F}$ ). In contrast, there was no difference between WT and Trem2 $2^{--}$mice fed a NCD (Supplemental Figure 3, C-G).

We performed unbiased transcriptome analysis of livers of WT versus Trem $2^{--}$mice after feeding mice a HFD for 8 weeks (31). In this screen, a cluster of 104 transcripts were upregulated and 57 were downregulated in response to Trem 2 deficiency (Supplemental Data Set 2 and Supplemental Figure 4A; GSE160022). Among these DEGs (Padj < 0.05), gene sets that contribute to fatty acid metabolic dysfunction, collagen fibril organization, and cytokine secretion were significantly enriched in Trem2 $2^{--}$livers, whereas those controlling long-chain/unsaturated fatty acid metabolism and monocarboxylic acid metabolism were overrepresented in WT livers (Figure 4, G and H). qPCR analysis in an independent cohort further confirmed that livers of HFD-fed Trem2 $2^{--}$mice had reduced expression of genes involved in the metabolic fates of polyunsaturated fatty acids (PUFAs), including long-chain fatty-acid CoA ligase 4 (Acsl4), steroid hormone metabolism (Hsd3b5), and hepatic trefoil factor 3 (Tff3). The expression of lipogenesis genes (Apoa4, CD36, and Cidea) and inflammatory cytokine genes (Ccl2, Ly6d, Cxcl10, and TNF- $\alpha$ ) were upregulated in Trem2 $2^{--}$livers (Supplemental Figure 4B). These data indicate that Trem 2 deficiency exacerbates the progression of NAFLD.

Last, we asked whether a defect of TREM2 impacted liver energetic metabolism and mitochondria function. Tandem mass spectrometry was first used to detect metabolites in both positive and negative ion modes in the livers of WT and Trem $2^{-/}$mice fed HFD for 8 weeks, after an overnight fasting. Totally, 219 and 201 lipids and lipid-like molecules were identified in the positive and negative ion modes, respectively (Supplemental Data Set 3). The levels of major fatty acid species, including ricinoleic acid (RA), linoleic acid (LA), dodecanedioic acid (DA), and arachidonic acid (AA), were more elevated in Trem $2^{--}$versus WT livers (Figure 4I and Supplemental Figure 4C, $P<0.05$ ). Acylcarnitine (AC) species ranging in size from 2 to 22 carbons were also analyzed, representing byproducts of substrate catabolism. Ions separating Trem2 $2^{--}$ and WT livers were identified as long-chain AC species (ranging from C14:0 to C22:0; Figure 4I, $P<0.05$ ), suggesting insufficient L-carnitine shuttle of long-chain fatty acids from the cytosol to the mitochondria in Trem2-- liver. In support of this, hepatic mitochondria in Trem2-- mice were less uniform and more fragmented compared with those in WT mice (Figure 4J). Moreover, mice lacking Trem 2 exhibited reduced ATP levels in the liver (Figure $4 \mathrm{~K})$. Together, these results suggested that Trem $2^{--}$liver is unable to appropriately adjust FAO and energy metabolism due to mitochondrial fragmentation after HFD.

TREM2 promotes macrophage-hepatocyte metabolic coordination both in vivo and in vitro. To explore whether the aggravation of hepatic steatosis in HFD-fed Trem 2-- mice is due to a defect of liver KCs, we depleted the KCs of HFD-fed WT and Trem2 $2^{-/}$mice by administration of gadolinium chloride ( $\mathrm{GdCl} 3$, Figure $5 \mathrm{~A}$, and ref. 32). Depletion of KCs was confirmed by a greater than $40 \%$ decrease in $\mathrm{F} 4 / 80^{+}$cells and the approximately $50 \%$ decrease in F4/80 mRNA expression in the liver (Supplemental Figure 5, A and B). Overall, compared with control mice, mice with KC depletion gained less body weight and had improved lipid metabolism, as evidenced by a decrease of serum and hepatic TG and cholesterol contents after 8 weeks of HFD (Figure 5, B and C, and Supplemental Figure 5C). Interestingly, in the $\mathrm{GdCl} 3$ group, Trem $2^{--}$and WT mice showed comparable liver and serum TG and cholesterol contents, liver fat accumulation, and ATP production (Figure 5, B-E). Together, these data support that Trem $2^{--}$liver KCs aggravate the development of hepatic steatosis.

To further define the beneficial roles of TREM2 in hepatic steatosis in vitro, bone marrow-derived macrophages (BMDMs) from WT or Trem $2^{-/}$mice were cocultured with primary hepatocytes isolated from WT or Trem2-- mice in trans-well plates (Figure $5 \mathrm{~F}$ ). Cultures were supplemented with palmitic acid (PA, 0.5 $\mathrm{mM}$ ) for 24 hours in serum-free conditions. A drastic increase in LDs was observed in both WT and Trem2- hepatocytes when cocultured with Trem $2^{--}$macrophages (Figure 5G). Under these conditions, a luciferase-based ATP assay showed that ATP production decreased in hepatocytes cocultured with Trem $2^{-1-}$ 
A

Trem2 $2^{-/-}$or littermate WT control Week of age

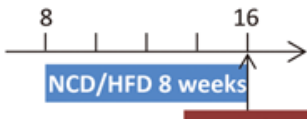

Sacrifice
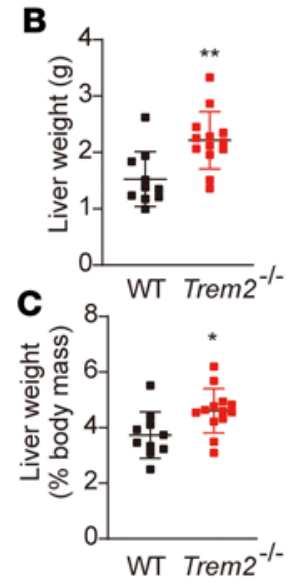

D

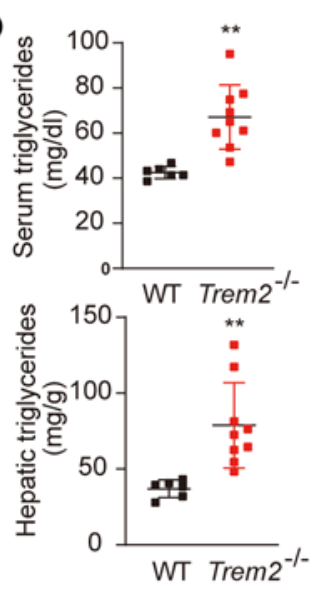

E
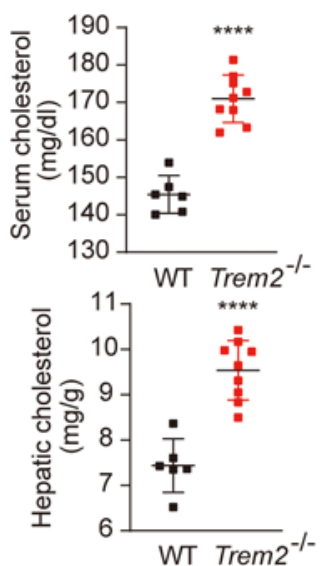

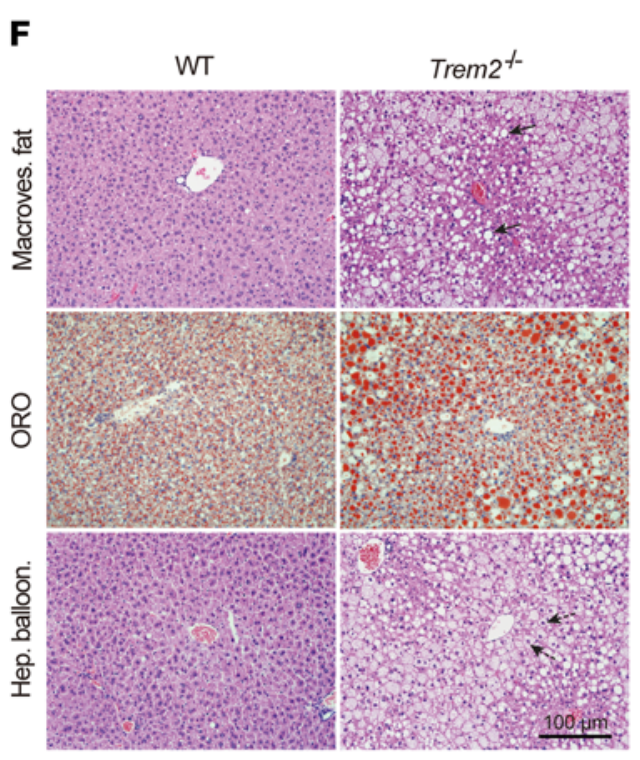

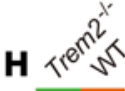

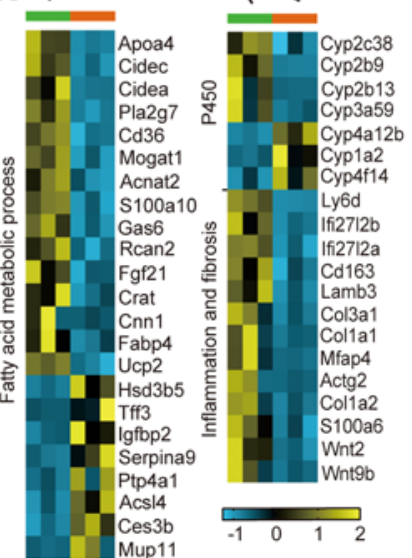

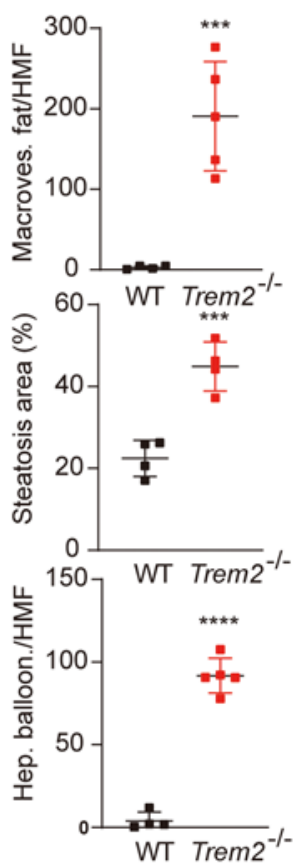

WT Trem2
G

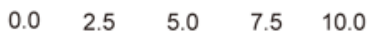

Fatty acid metabolic process Collagen fibril organization

Vesicle-mediated transport Leukocyte migration Fibroblast proliferation Interleukin-6 production Response to superoxide Macrophage cytokine production Epoxygenase P450 pathway Acute-phase response Interleukin-1 secretion
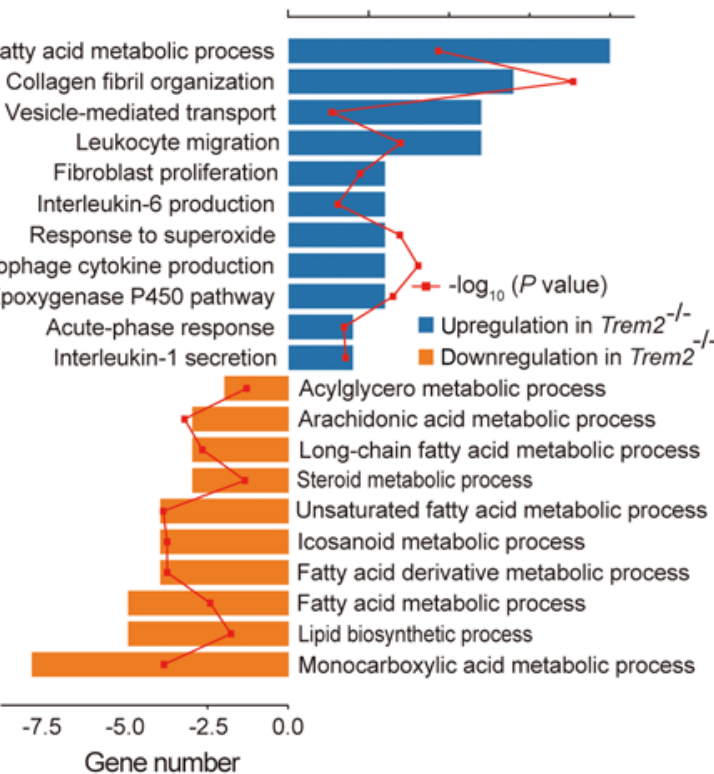

Gene number



J

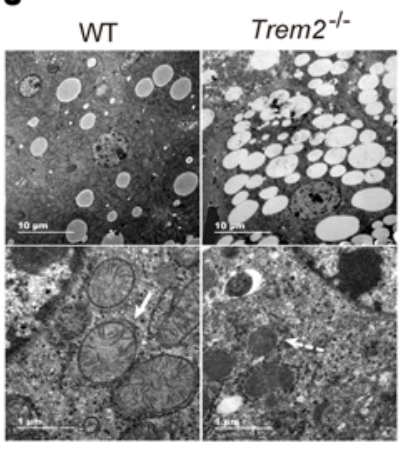

K

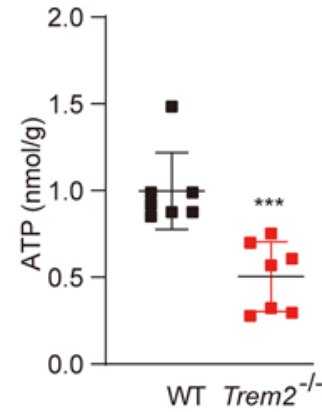


Figure 4. Trem2 deficiency exacerbates hepatocyte mitochondrial dysfunction and accelerates NAFLD progression in a mouse model. (A) Experimental design used to evaluate the role of Trem2 in NAFLD progression. Liver weights (B) and liver weight/body weight ratios (C) of WT and Trem2 $2^{-1-}$ mice after being fed HFD for 8 weeks. $n=10$ in WT group; $n=13$ in Trem2 ${ }^{-1-}$ group. Levels of TG (D) and cholesterol (E) in serum and livers from mice after being fed HFD for 8 weeks. $n=6$ in WT group; $n=9$ in Trem2 $2^{-1-}$ group. (F) Representative images of H\&E- and ORO-stained liver sections. $n=4$ in WT group; $n=4-5$ in Trem2 $2^{-1-}$ group. Solid and dashed black arrows indicate hepatocytes with macrovesicular fat (Macroves. fat) or hepatocyte ballooning (Hep. balloon.), respectively. Number of hepatocytes with Macroves. fat or Hep. balloon. and percentage of ORO-positive area per high magnification field (HMF) was determined by Imagel from 6 fields per section. Scale bar: $100 \mu \mathrm{m}$. (G) GO analysis of the identified DEGs between WT and Trem $2^{-/-}$groups ( $n=3$ per group). (H) Heatmap showing the expression pattern of the identified DECs ( $n=3$ per group). The color key indicates the expression levels. (I) Heatmap displays the upregulated and downregulated lipid species in livers ( $n=9$ mice per group), $P$ $<0.05$. The color key indicates the lipid levels. (J) Representative TEM images of mouse hepatocytes (upper) and hepatocellular mitochondria (bottom). $n=$ 4 in WT group; $n=5$ in Trem2 $2^{-1-}$ group. Solid and dashed white arrows refer to complete and fragmented mitochondria, respectively. Scale bars: $10 \mu \mathrm{m}$ and 1 $\mu \mathrm{m}$, respectively. (K) ATP levels in WT and Trem2 $2^{---}$livers. $n=7$ per group. All data are shown as the mean \pm SD. The data were analyzed by an unpaired, 2-tailed Student's $t$ test. ${ }^{*} P<0.05,{ }^{* *} P<0.01,{ }^{* *} P<0.001$, ${ }^{* * *} P<0.0001$

macrophages compared with the hepatocytes cocultured with WT BMDMs (Figure 5H). These results support that TREM2 deficiency impairs macrophage-hepatocyte metabolic coordination in a fatty acid-enriched microenvironment.

Trem 2 deletion leads to changes in macrophage-Exos numbers and content. To explore the potential mechanism underlying FAO defects in livers from Trem $2^{-/-}$mice fed HFD for 8 weeks, we profiled KC transcriptomes isolated from these mice and their WT littermates by RNA-seq (Supplemental Data Set 4; GSE160022). Gene ontology (GO) analysis revealed that antigen processing and presentation, mitochondrial oxidative phosphorylation, and ATP synthesis were impaired in Trem $2^{-/-}$KCs. However, genes linked to chemotaxis and inflammation (Ccr2, Cx3cr1, Spp1) were upregulated in Trem2 $2^{-1-}$ KCs (Figure 6A and Supplemental Figure 6A). Interestingly, genes involved in Exos secretion (Cd63, Cd9) were enriched in Trem2 $2^{-/-}$KCs (Supplemental Figure 6A).

Macrophage-derived Exos can be taken up into hepatocytes to modulate the function of recipient cells (33). Thus, we conducted in vitro studies to confirm whether Trem $2^{-/-}$macrophage-derived Exos were the underlying mechanism of Trem $2^{-/-}$liver FAO defects and ATP deficiency. BMDMs with different genotypes were stimulated with PA $(0.5 \mathrm{mM})$ or control vehicle for 12 hours. After removal of dead cells and debris from the conditioned medium, extracellular vesicles (EVs) were isolated by differential ultracentrifugation. These EVs were confirmed to primarily consist of Exos, as evidenced by the expression of the Exos-specific protein markers CD63, CD81, and Alix, but not cytochrome C (mitochondria), GM-130 (Golgi), or calnexin (ER) proteins (Supplemental Figure 6B). TEM (Figure 6B) and NanoSight analysis (Figure 6C) corroborated that the particles were BMDM-derived Exos (BMDM-Exos) with a diameter of 30-150 nm. The overall Exos number isolated from the same amount of medium from the Trem $2^{-/-}$BMDMs group was much higher than that from WT BMDMs group (Figure 6C).

Next, we incubated the WT primary hepatocytes with equal amounts of WT or Trem $2^{-/-}$BMDM-Exos $(20 \mu \mathrm{g} / \mathrm{mL})$ for 12 hours under PA (0.5 mM) stimulation. Compared with WT BMDM-Exostreated hepatocytes, hepatocytes incubated with Trem2 $2^{-/}$BMDMExos showed a significant increase in LD accumulation (Figure 6D), suggesting that the composition of WT BMDM-Exos and Trem2-1BMDM-Exos diverges under a fatty acid-rich environment. Further, to replicate the in vitro phenomenon in vivo, WT mice fed a HFD for 7 weeks were used as recipients and intravenously injected with PKH26-labeled WT or Trem2 $2^{-/}$BMDM-Exos for 3 weeks $(30 \mu \mathrm{g}$ every 7 days, Figure 6E). The appearance of red fluorescent PKH26 dye in the livers of recipient mice indicated the in vivo uptake of Exos in the liver (Supplemental Figure 6C). Compared with the WT BMDM-Exos group, Trem $2^{--}$BMDM-Exos-injected mice gained more liver weight and liver weight-to-body weight ratios, while the body weight and epididymal fat pad weights were comparable between the 2 groups (Figure 6F and Supplemental Figure 6, $\mathrm{D}$ and E). Trem2 $2^{-/}$BMDM-Exos-injected mice showed increased serum and liver TG content and hepatic LD accumulation (Figure 6, G-I). ATP production was lower in the livers of Trem2 $2^{--}$BMDMExos-injected mice compared with WT BMDM-Exos-injected mice (Figure 6J). Therefore, our data show that liver macrophage TREM2 attenuates NAFLD progression in an Exos-dependent manner.

Macrophage-derived Exos impair hepatocyte mitochondria due to high content of miR-106b-5p that blocks Mfn2. miRNAs are regulatory molecules that can be packaged into Exos and secreted from cells (33-35). To assess the NAFLD-induced changes in expression of KCs miRNAs, we conducted deep sequencing of small RNAs from KCs isolated from WT and Trem $2^{-/-}$mice fed HFD for 8 weeks. More than 1000 miRNAs were identified in KCs (Supplemental Data Set 5; GSE160022). A set of miRNAs were upregulated in Trem $2^{-/-}$versus WT KCs, among which miR-106b-5p was the most abundant miRNA in KCs (Figure 7A, Supplemental Data Set 5, and ref. 36). In an independent cohort, our results confirmed that KCs from Trem $2^{-/-}$mice expressed greater intracellular levels of miR-106b-5p after 8 weeks of HFD feeding (Supplemental Figure 7A), which led to increased levels of miR-106b-5p present in the livers (Supplemental Figure 7B). Consistent with these in vivo results, the miR-106b-5p abundance was significantly increased in Trem2 $2^{-/}$BMDM-Exos ( 6-fold) after treatment with PA (0.5 mM) for 12 hours (Supplemental Figure 7C).

To evaluate the role of miR-106b-5p on lipid metabolism, we transfected BMDMs with Cy3-labeled miR-106b-5p mimic or negative control (NC) via lipofectamine RNAiMAX (Supplemental Figure 7, D and E). After 12 hours of coculture with miR-106b$5 \mathrm{p}$ mimic-transfected BMDMs, the hepatocyte exhibited efficient uptake of the macrophage-secreted miR-106b-5p ( 80\% efficien$\mathrm{cy}$ ), as indicated by the presence of red fluorescence (Supplemental Figure 7D) and increased miR-106b-5p abundance within the hepatocytes (Supplemental Figure 7F). The increased miR-106b$5 p$ abundance in hepatocytes led to a significant increase in LD accumulation after PA treatment for 12 hours in serum-free conditions, accompanied by decreased ATP production within the hepatocytes (Figure 7, B and C).

miR-106b-5p was previously reported to target Mfn2 (37). Mfn2 plays a crucial role in controlling mitochondrial fusion, which is required for oxidative phosphorylation, mitochondrial DNA biogenesis, mitophagy regulation, and metabolic adaptation (38). Since Mfn2 ablation in the liver was shown to cause a NASH-like 
A

Trem2 $2^{\text {t- }}$ or littermate WT control



Week of age

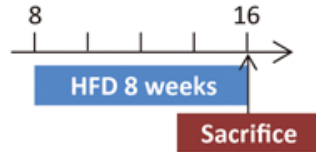

B

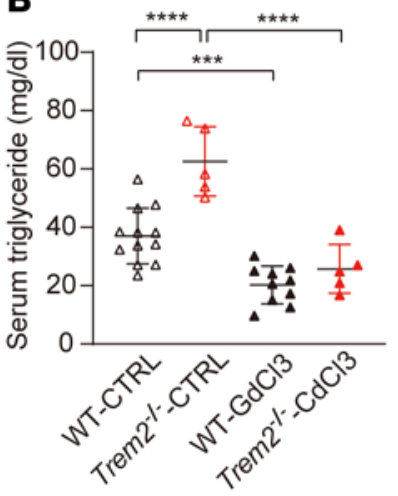

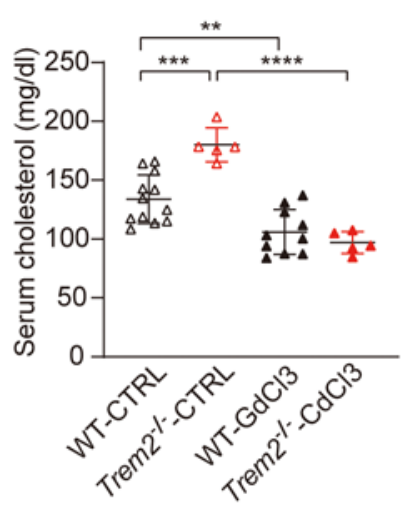

WT (ORO)
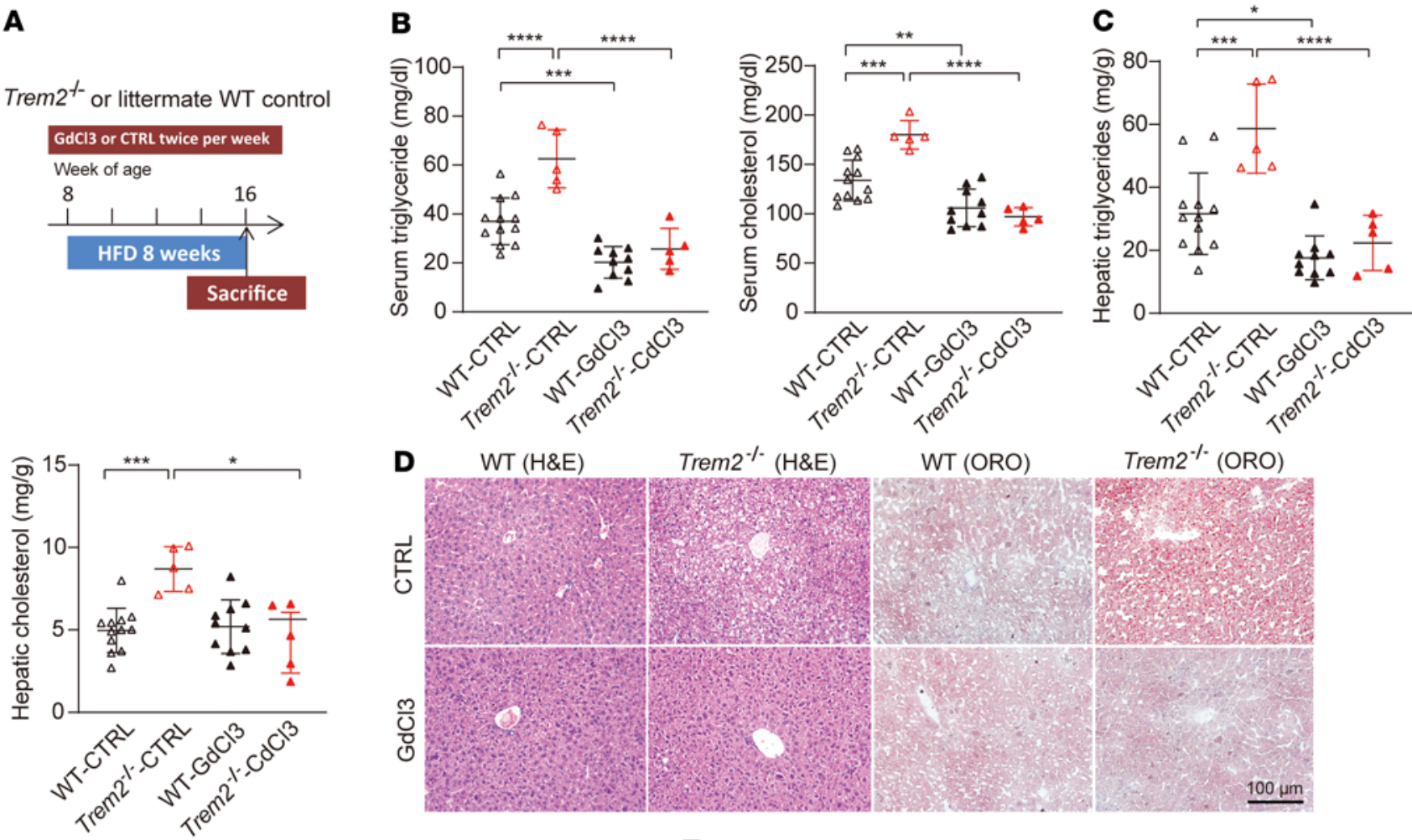

D WT (H\&E) Trem2\% $2^{\%}$ H\&E)

Trem2 $^{\%}$ (ORO)


Figure 5. Macrophage Trem2 deficiency exacerbates hepatocyte lipid accumulation both in vivo and in vitro. (A) Schematic representation of the timing

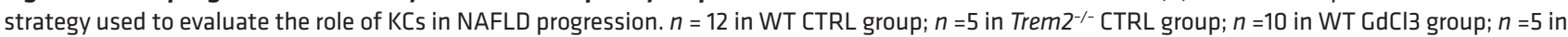
Trem2 $2^{-1-} \mathrm{CdCl} 3$ group. (B and C) Serum and liver triglyceride or cholesterol levels in response to KCs depletion. (D) Representative images of H\&E- and OROstained liver sections. $\mathrm{H} \& \mathrm{E}$ reveals tissue composition and macrovesicular fat, and ORO visualizes lipid droplets. Number of hepatocytes with macrovesicular fat and percentage of ORO-positive area per HMF was determined by Image from 6 fields per section. Scale bar: $100 \mu \mathrm{m}$. (E) ATP contents in the liver. (F and $\mathbf{G})$ Representative image of ORO-stained hepatocytes after coculture with BMDMs (F). BMDMs from WT or Trem2 ${ }^{-1-}$ mice were cocultured with primary hepatocytes isolated from WT or Trem2 $2^{-1-}$ mice in transwell plates. Cultures were added with PA (0.5 mM) for 24 hours in serum-free conditions. 
Lipid accumulation in hepatocytes was determined by quantification of ORO-positive area as a percentage of whole image area by ImageJ (C). $n=9$ per group. Scale bar: $50 \mu \mathrm{m}$. (H) ATP content in hepatocytes was quantified by luciferase assay. $n=9$ per group. The data were analyzed by 1-way analysis of variance with Bonferroni corrections for multiple comparisons. All data are shown as the mean $\pm \mathrm{SD}$. ${ }^{*} P<0.05$, ${ }^{* *} P<0.01$, ${ }^{* *} P<0.001$, **** $P<0.0001$

phenotype and liver cancer $(39,40)$, we examined whether the upregulated miR-106b-5p downregulates Mfn2 protein levels and causes mitochondrial dysfunction in our model. BNL.CL2 hepatocytes were transfected with the indicated miR-106b-5p mimic or NC and examined by immunoblotting. As shown in Figure 7D, overexpression of miR-106b-5p markedly downregulated Mfn2 protein levels in hepatocytes. Taken together, Trem $2^{-/-}$macrophages exacerbate hepatocyte FAO dysfunction by promoting macrophage release of Exos that impair hepatocyte mitochondrial homeostasis through their content of miR-106b-5p, which blocks Mfn2.

Trem 2 deficiency increases the susceptibility to NAFLD-associated sepsis in vivo. To further assess the involvement of TREM2 in the susceptibility of NAFLD mice to sepsis, we performed a survival study on WT and Trem2 $2^{-/}$mice fed a NCD or HFD for 8 weeks. After dietary intervention, lethal polymicrobial sepsis was induced by cecal ligation and puncture (CLP), and mice were monitored for 3-day survival (Figure 8A). We found that all of the HFD-fed mice (WT and Trem2 $2^{-/}$) died within 3 days following the surgery, whereas less than $40 \%$ of NCD-fed mice (WT and Trem $2^{--}$) died within the 3-day study period (Figure 8B). Compared with WT NAFLD mice, Trem $2^{-/-}$NAFLD mice had a shorter survival time following induction of lethal sepsis (Figure 8B).

To explore the factors that exacerbate septic mortality, systemic bacterial burden and cytokines were measured. Compared with HFD-fed WT mice, the systemic bacteria burden was increased in HFD-fed Trem $2^{-/-}$mice after sepsis insult; however, there was no difference between the NCD-fed Trem $2^{-/-}$mice and HFD-fed Trem $2^{-/-}$mice (Figure $8 C$ ). Additionally, there was no significant difference in cytokine production among different mouse genotypes either with NCD or HFD treatment (Figure 8D). Given the difference in the survival between the HFD-fed Trem $2^{-/-}$group and the other 3 groups, our data suggested that factors other than bacterial burden and proinflammatory cytokines worsen the sepsis outcome in HFD-fed Trem $2^{-1-}$ mice.

Furthermore, TEM showed more severe mitochondrial fragmentation in Trem $2^{-/-}$livers than in WT livers, accompanied by a significant decrease in ATP production (Figure 8, E and F), indicating that the fragility of HFD Trem $2^{-/-}$liver may be an important risk factor in sepsis insult. In support of this possibility, more severe liver injury and lung injury were observed in Trem $2^{-1-}$ than that in WT mice on day 1 after CLP insult, as evaluated by bleeding and inflammatory cell infiltration (Supplemental Figure 8A). The plasma ALT concentration of HFD-fed Trem2 $2^{-/-}$mice was approximately double that in HFD-fed WT mice (Supplemental Figure $8 \mathrm{~B})$. In addition, there was about $50 \%$ decrease in plasma albumin concentration in the HFD-fed Trem $2^{-/-}$mice (Supplemental Figure 8C), indicating that liver failure occurred in HFD-fed Trem $2^{-/-}$mice.

An additional LPS-induced sepsis model (10 mg/kg LPS) was performed in NCD-fed Trem $2^{-/-}$and WT littermates. NCD-fed
Trem $2^{-/-}$mice showed comparable survival and organ injury to the NCD-fed WT mice (Supplemental Figure 8, D-F), corroborating that TREM2 deficiency increases the susceptibility to septic insult only in the presence of a liver metabolic disorder caused by HFD. Taken together, our results support the notion that liver metabolic failure and severe liver dysfunction are important causes of septic death in HFD-fed Trem $2^{-1-}$ mice. We do not exclude that defects in the Trem $2^{-1-}$ macrophages of other organs may also contribute to septic death.

Elevated TREM2 gene dosage in liver macrophages suppresses steatohepatitis and relieves sepsis-induced organ dysfunction. To further corroborate that macrophage TREM2 alleviates altered hepatic energetic metabolism and subsequent liver dysfunction in NAFLD-associated sepsis, we tested whether increasing the macrophage TREM2 gene dosage could modify disease pathogenesis. We generated a transgenic mouse line expressing the common variant of human TREM2 (BAC-TREM2) in addition to WT endogenous TREM2. Because human and mouse TREM2 are highly homologous and have evolutionarily conserved functions, BAC-TREM2 mice provide a model of TREM2 overexpression (41, 42). BAC-TREM2 and WT littermates were placed on HFD for 10 weeks (Figure 9A). To verify cell type-specific expression of human TREM2 transgene in livers of mice fed HFD for 10 weeks, we performed immunofluorescence microscopy of liver sections using an antibody against the $\mathrm{C}$ terminus of human TREM2. Human TREM2 colocalized exclusively with the macrophage marker Iba1 (Supplemental Figure 9A). In parallel, real-time qPCR data showed BAC-TREM2 mice expressed murine Trem 2 transcripts at levels comparable to WT mice after 10 weeks of HFD (Supplemental Figure 9B). Upon HFD feeding, BAC-TREM2 mice featured decelerated body/liver weight gain (Figure 9, B and C and Supplemental Figure 9C). No difference was detected in white adipose tissue weight between the BAC-TREM2 mice and WT littermates (Supplemental Figure 9D). H\&E and ORO analysis of liver sections showed that BAC-TREM2 mice had alleviated macrovesicular fat content, ballooning degeneration and lobular inflammation, and less LD accumulation after 10 weeks of HFD (Figure 9, D-F). Consistently, BAC-TREM2 mice showed improved hepatic FAO after overnight fasting, as evidenced by decreased hepatic TG content and increased ATP production (Figure 9, G and H).

To determine whether the elevated macrophage TREM2 dosage could impact susceptibility of NAFLD mice to polymicrobial sepsis-induced liver injury, sepsis was induced by mild CLP and mice were monitored for organ injuries, systemic bacteria burden, and cytokine production. Liver injury and lung injury were alleviated in the BAC-TREM2 group compared with WT littermates, accompanied by low blood bacteria burden and improved hepatic ATP production (Figure 9, I-K). There was no difference in systemic cytokine production, except for a trend in MCP-1 reduction in the BAC-TREM2 group (Supplemental Figure 9E). Overall, our data suggest that TREM2 overexpression in liver macrophages plays a protective role in defense against NAFLD-associated sepsis by facilitating liver switch to FAO metabolism.

\section{Discussion}

The liver is a prime target in sepsis-related injury, especially in the context of preexisting hepatic impairment (43). Here, we performed a clinical cohort study and showed that NAFLD is an independent 
A

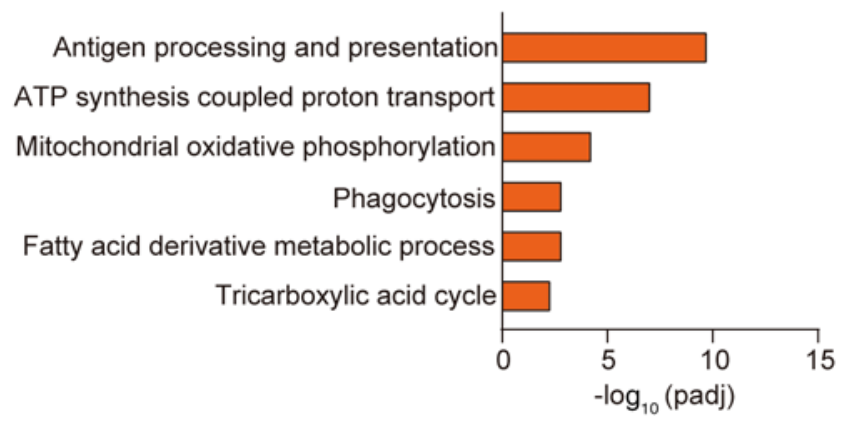

Upregulation in Trem2-\% $\mathrm{KCs}$

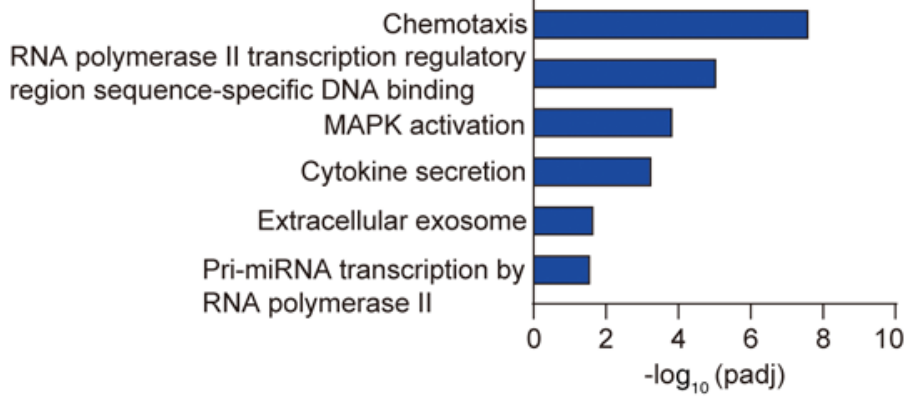

B
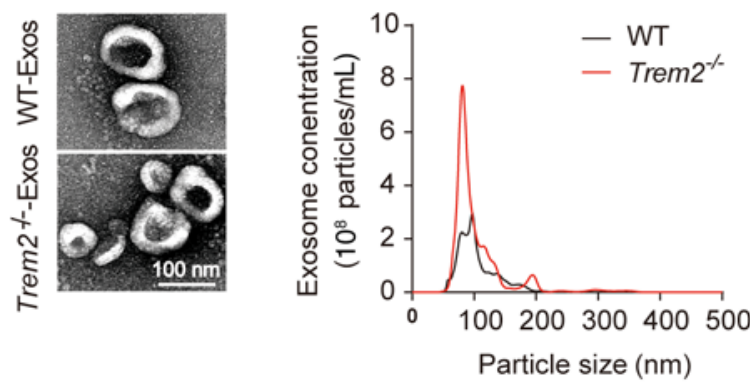

D BMDMs Primary hepatocyte (WT) (WT or Trem2 $2^{-/-}$


E



\section{F}
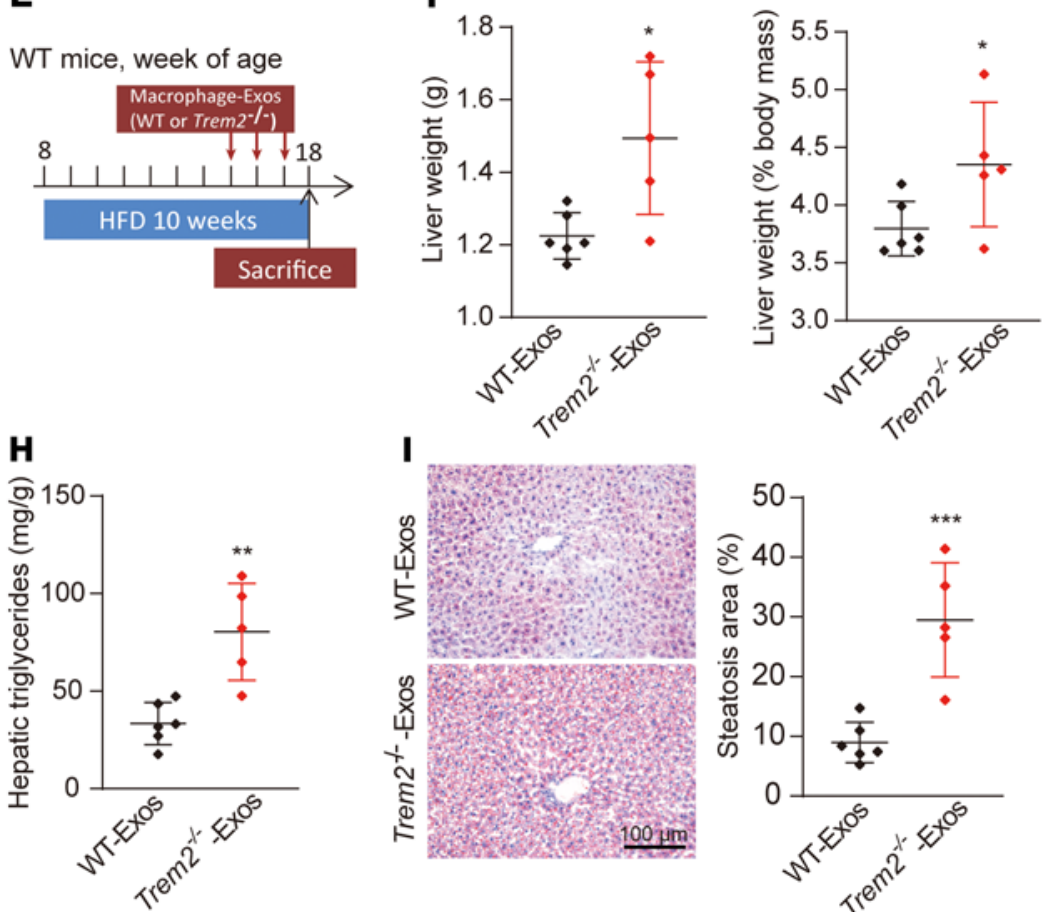

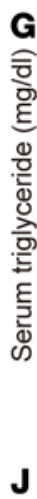

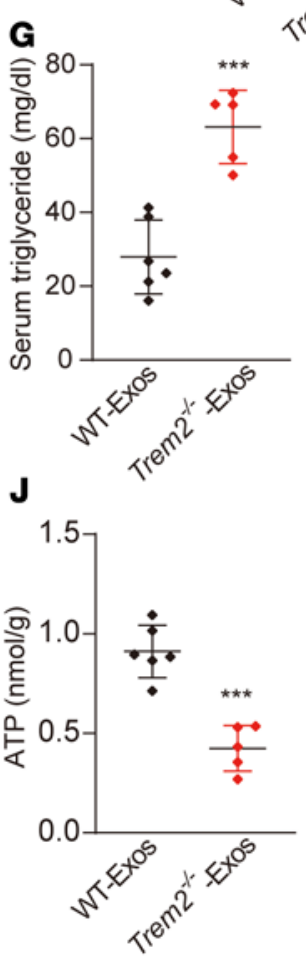

Figure 6. Trem2 deletion changes macrophage-Exos numbers and contents. (A) GO analysis of DEGs in KCs from WT or Trem2-/- mice fed HFD for 8 weeks. $n=3$ per group. (B) Representative TEM images of Exos from WT and Trem2 $2^{-1-}$ BMDMs incubated with PA (0.5 mM) for 12 hours. Scale bar: $100 \mathrm{~nm}$. (C) Representative results of nanoparticle tracking analysis demonstrate size distribution of Exos derived from WT and Trem2 $2^{-1-}$ BMDMs. $n=5$ per group. (D) WT primary hepatocytes were incubated with WT or Trem2-/- BMDM-derived Exos (20 $\mu \mathrm{g} / \mathrm{mL})$ and PA (0.5 mM) for 12 hours. Lipid accumulation in hepatocytes was determined by quantification of ORO-positive area as a percentage of whole image area by Image). $n=16$ in WT group; $n=19$ in Trem2 $2^{-1-}$ group. Scale bar: $25 \mu \mathrm{m}$. (E) Experimental design used to evaluate BMDM-Exos in NAFLD progression. $n=6$ in WT-Exos group; $n=5$ in Trem2 $\%$-Exos group. (F) Liver weights and liver weight/body weight ratios of mice with Exos treatment. (G and $\mathbf{H})$ Serum and liver triglyceride levels in response to Exos treatment. (I) Representative images of ORO-stained liver sections from mice treated with Exos from WT or Trem2 ${ }^{-/-}$BMDMs. Scale bar: $100 \mu \mathrm{m}$. Percentage of ORO-positive area per HMF were determined by Imagel from 6 fields per section. (J) ATP content in hepatocytes was quantified by luciferase assay. Data are presented as the mean $\pm \mathrm{SD}$. The data were analyzed by an unpaired, 2-tailed Student's $t$ test. ${ }^{*} P<0.05,{ }^{* *} P<0.01,{ }^{* * *} P<0.001,{ }^{* * * *} P<0.0001$ 
A

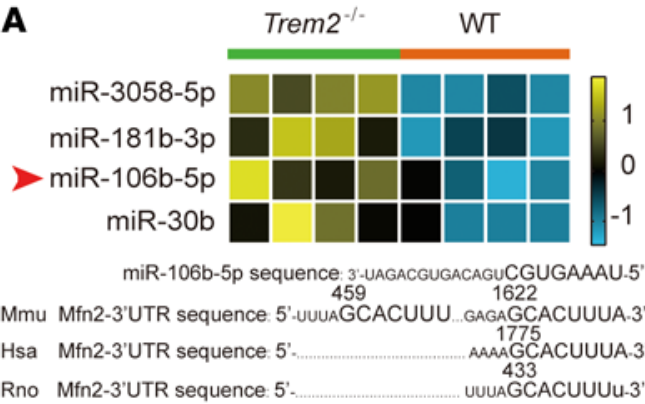

B
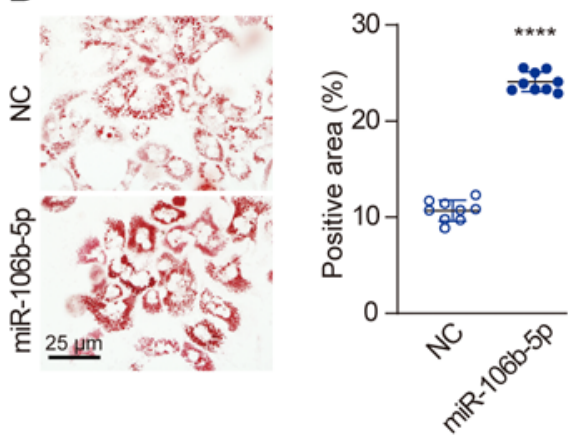

C
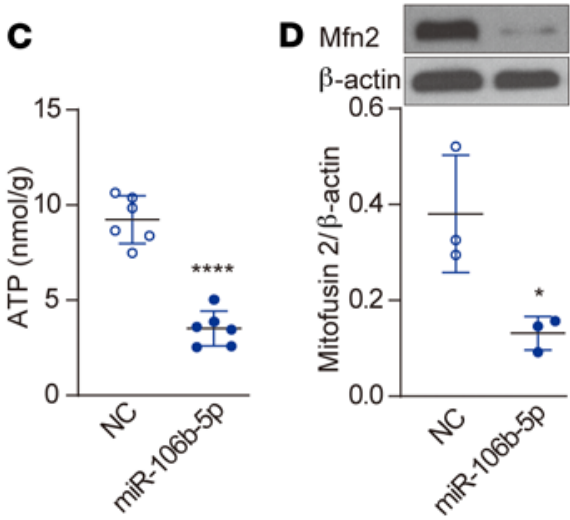

Figure 7. Trem2 deletion impacts macrophage-Exos miRNA profiles. (A) Heatmap of small RNA transcripts in WT and Trem2-1- KCs after 8-week HFD and sequence alignment of miR-106b-5p with $3^{\prime}$ UTRs of mouse (Mmu), human (Hsa), and rat (Rno) Mfn2. $n=4$ for each group. The color key indicates the expression levels. (B and C) WT BMDMs were transfected with either NC or miR-106b-5p mimic and then cocultured with primary WT hepatocytes in a transwell plate for 12 hours with $0.5 \mathrm{mM}$ PA stimulation. Lipid accumulation in hepatocytes was determined by quantification of ORO-positive area as a percentage of whole image area by Imagej (B), $n=$ 9 per group. Scale bar: $25 \mu \mathrm{m}$. ATP content in hepatocytes was quantified by luciferase assay (C), $n=6$ per group. (D) Representative Western blot images of Mfn2 expression in BNL CL.2 cells, which were transfected with either NC or miR-106b-5p mimic and then stimulated with $0.5 \mathrm{mM} \mathrm{PA}$ for 6 hours. The integrated density of the blots was analyzed by Image. $n=3$ per group. Data are presented as the mean \pm SD. Data were analyzed by an unpaired, 2-tailed Student's $t$ test. ${ }^{*} P<0.05$, ${ }^{* * * *} P<0.0001$.

risk factor for hospital mortality after adjusting for age, sex, BMI, and site of infection. Liver transcriptome analysis and liver biopsies showed that mortality in NAFLD-associated sepsis was associated with impaired lipid metabolism, severe hepatic mitochondrial fragmentation, and decreased ATP content. Remarkably, we found that NAFLD was paralleled by the expansion of liver macrophages expressing TREM2, which impacted hepatic mitochondrial dysfunction. Trem2-deficient macrophages released Exos that impaired hepatocytic mitochondrial structure and energy supply because of their high content of miR-106b-5p, which blocks Mfn2. Trem2 deletion exacerbated NAFLD as well as susceptibility of mice to sepsis. Conversely, overexpression of TREM2 in liver macrophages attenuated steatohepatitis and sepsis-induced mortality. Collectively, these findings support hepatic mitochondrial dysfunction as a significant mediator of the pathogenesis of NAFLD and NAFLD-associated sepsis, and demonstrate a role for macrophage TREM2 in protecting hepatocytes against mitochondrial dysfunction (Figure 10).

Frequently, septic patients exhibit chronic diseases such as NAFLD, which is the most common chronic liver disease $(4,5)$. In our current prospective clinical cohort study, $24.62 \%$ patients with sepsis were identified as NAFLD. The NAFLD cohort showed significantly increased 28-day mortality and hospital mortality compared with the non-NAFLD cohort. Moreover, within the NAFLD cohort, female patients and patients aged 65 and over had a predominantly higher risk for adverse clinical outcomes following sepsis than patients in the other cohorts, probably due to the high rate of comorbidities in those patients. Diabetes is known to strongly associate with NAFLD, but whether this may lead to adverse clinical outcomes was unknown (20). We found that both the 28-day mortality and hospital mortality in the NAFLD group were still significantly higher than the non-NAFLD group after excluding these diabetic patients. The same tendency was also observed in diabetic patients. Moreover, in the NAFLD group, the mortality in patients with and without diabetes was comparable, indicating a strong link between NAFLD and septic mortality. Since sepsis is associated with a shift from glycolysis to FAO for energy supply in the liver (22), lipid overload and excess of toxic fatty acids in NAFLD may impair mitochondrial FAO, affecting liver function and, consequently, energy supply to other vital organs during sepsis. Thus, targeting hepatic FAO and energy metabolism may improve the outcome of septic patients with NAFLD.

A recent study identified the emergence of $T r e m 2^{+} \mathrm{NASH}-$ associated KCs as a feature of mouse and human NASH (29). In our study, liver immunostaining also revealed increased numbers of CD68 ${ }^{+}{ }^{+} R E M 2^{+}$and F4 $/ 80^{+}$TREM $2^{+} \mathrm{KCs}$ in NAFLD donors and HFD-fed mice, respectively. Since it was shown that monocytes can acquire a KC profile once they reach the space of Disse (44), whether TREM $2^{+} \mathrm{KCs}$ in NAFLD livers are bona fide KCs or derive from the infiltration and differentiation of monocytes remains unclear. TREM2 was recently shown to control lipid metabolism in microglia and adipose tissue macrophages $(12,13,30)$. Our study extends these findings, showing that deletion of Trem 2 in a mouse NAFLD model increased hepatocyte lipid content and accumulation of fatty acid species, reduced long-chain acylcarnitine species and ATP production, and augmented mitochondria fragmentation. Moreover, Trem $2^{-/}$NAFLD mice showed worse outcomes than WT NAFLD mice following polymicrobial sepsis, whereas overexpression of TREM2 on KCs alleviated NAFLD and adverse septic outcome. While the impact of TREM2 on sepsis outcome may be due to its influence on hepatocyte energy supply, FAO, and mitochondrial function, we cannot exclude additional contributions of TREM2 to other macrophage compartments involved in immune responses. 
A

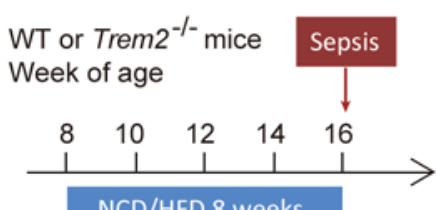

NCD/HFD 8 weeks
B

$$
\begin{aligned}
& - \text { WT NCD } \\
& - \text { Trem2 } 2^{-/-} \text {NCD }
\end{aligned}
$$



E



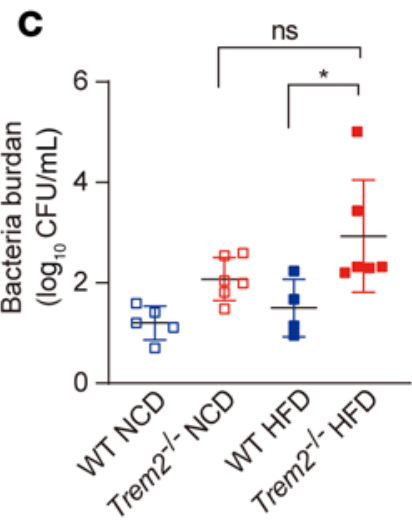

$\mathbf{F}$

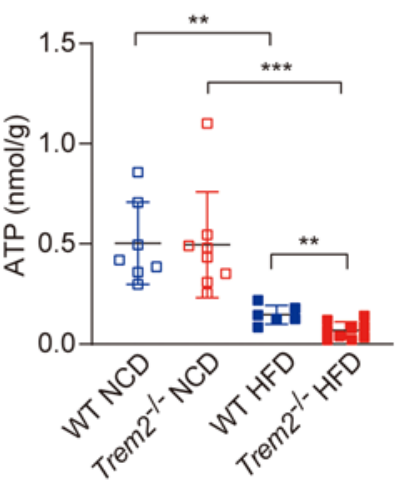

Figure 8. Trem2 deficiency increases susceptibility to NAFLD-associated sepsis. (A) Experimental design used to set up NAFLD and sepsis. (B) WT and Trem2 $2^{-1-}$ mice fed NCD or HFD for 8 weeks were subjected to lethal CLP with an 18-gauge needle, and mice survival was monitored every 12 hours. $n=10$ for NCD WT mice; $n=11$ for NCD Trem2 $2^{-1-}$ mice; $n=11$ for HFD WT mice; $n=13$ for HFD Trem2 $2^{-1-}$ mice. (C) Blood bacterial burden at 24 hours post-CLP. $n=5$ for NCD WT mice; $n=6$ for NCD Trem2 $2^{--}$mice; $n=4$ for HFD WT mice; $n=6$ for HFD Trem2 $2^{-1-}$ mice. (D) Plasma cytokine markers were quantified 24 hours after CLP. $n=5$ per group. (E) Representative TEM images of mouse hepatocellular mitochondria obtained from WT or Trem2 $2^{-1-}$ mice livers 24 hours after CLP. $n=2-6$ for each group. Scale bar: $1 \mu \mathrm{m}$. (F) Levels of ATP contents in livers from WT or Trem2 $2^{-1-}$ mice at 24 hours after CLP. $n=7$ in WT NCD group; $n=8$ in Trem $2^{-1-}$ NCD group; $n=6$ in WT HFD group; $n=9$ in Trem2 $2^{-1-}$ HFD group. Data are presented as the mean \pm SD. Data were analyzed by 1-way analysis of variance with Bonferroni corrections for multiple comparisons (C, $\mathbf{D}$, and $\mathbf{F})$. The survival rates were analyzed by log-rank test (B). ${ }^{*} P<0.05,{ }^{* *} P<0.01,{ }^{* * *} P<0.001$.

The critical role of macrophage TREM2 in attenuating lipid accumulation in hepatocytes in the NAFLD model was corroborated by an in vivo KC depletion model and in vitro coculture system. Since TREM2 is expressed in macrophages but not hepatocytes, how could TREM2 in liver KCs control lipid metabolism in hepatocytes? We found that the role of macrophage TREM2 in hepatic lipid handling and catabolism was related to the Exos pathway: Trem2macrophages released more Exos than WT macrophages, and Exos were taken up by neighboring hepatocytes modulating their function through miRNAs (33). Among several miRNAs, we focused on miR-106b-5p because it was enriched in KCs, was upregulated in obese adipose tissue macrophage-derived Exos $(33,36)$, and was shown to target the mitochondrial membrane protein Mfn2, which is important for mitochondria function $(37,39)$. In a transwell assay, Cy3-labeled miR-106b-5p transfected in macrophages was readily detected in hepatocytes with hundreds of fold increase in expression. This increase in hepatocyte abundance of miR-106b-5p led to a significant increase in lipid droplet accumulation after PA treatment, accompanied by decreased ATP production and Mfn2 downregulation. Understanding how TREM2 controls Exos secretion and miR-106b-5p expression will require additional studies.
In conclusion, by examining a large cohort of patients with NAFLD and sepsis, we defined the contribution of NAFLD to sepsis mortality. Our findings underscore the importance of mitochondrial metabolic dysfunction in the pathogenesis of NAFLD-associated sepsis, show that metabolic coordination between liver macrophages and hepatocytes protects from NAFLD, demonstrate the requirement for TREM2 for such coordination, and highlight the TREM2 pathway as a potential avenue of therapeutic intervention for NAFLD-associated sepsis.

\section{Methods}

Study design, setting, and population. Patients who met the Sepsis 3.0 clinical criteria were screened for eligibility within the first 24 hours after they were admitted to the intensive care unit (ICU) of Zhejiang University Hospital between September 2012 and January $2019(1,45)$. Information was extracted from a prospectively designed database. A total of 524 patients who had CT of the abdomen within 2 months prior to ICU admission or during ICU stay were enrolled. Exclusion criteria were age younger than 18 years or older than 80 years, with liver cirrhosis, with alcohol consumption ( $>140 \mathrm{~g} /$ week in men or $>70$ $\mathrm{g} /$ week in women), with a previous positive test for hepatitis B surface 
A

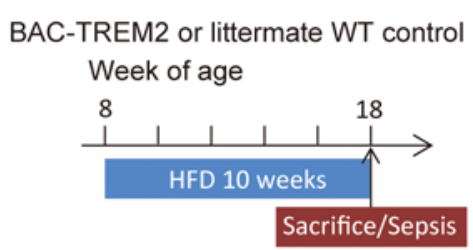

E
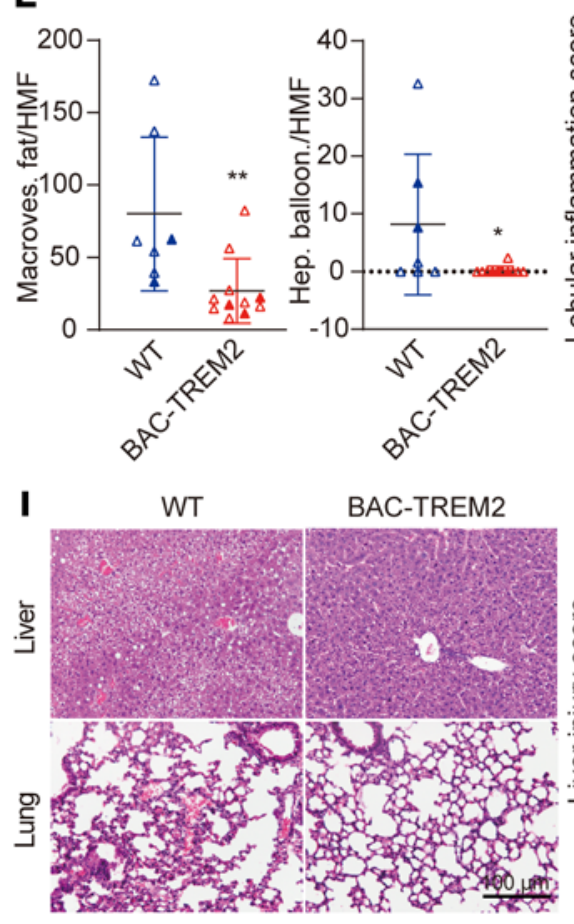

B



C
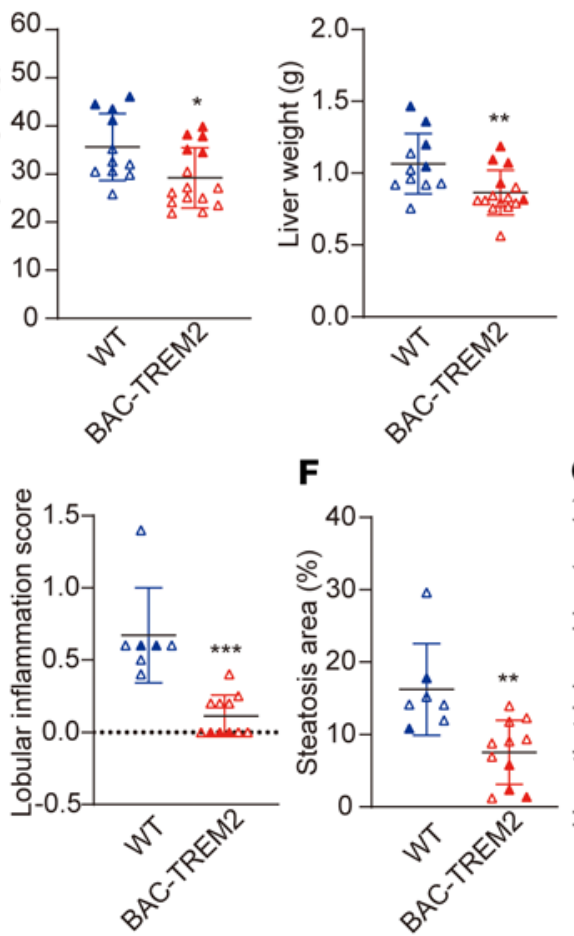

F
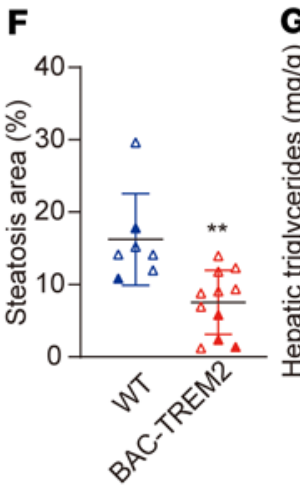

D

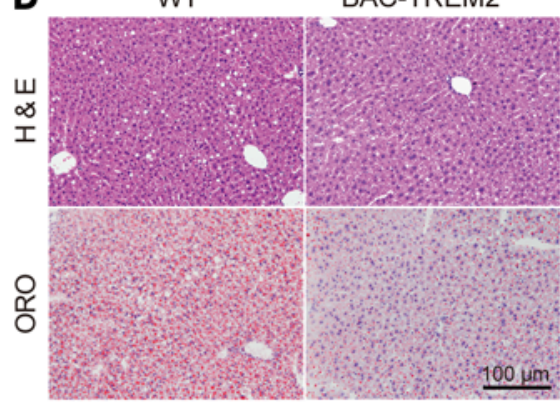

G

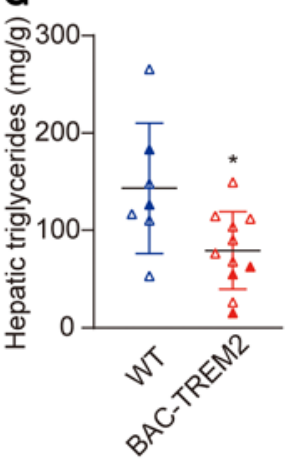

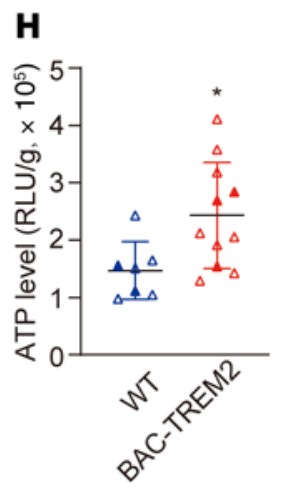
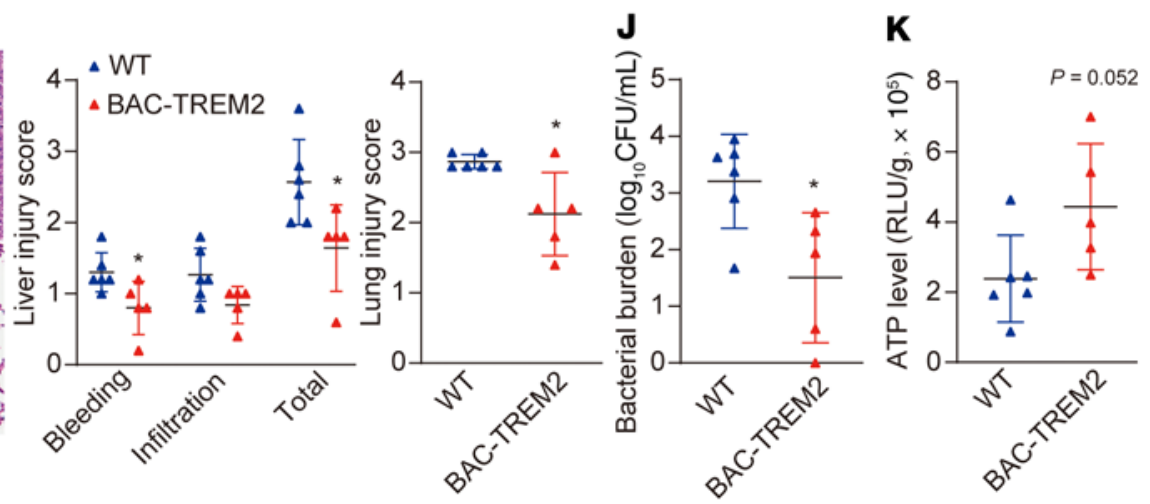

Figure 9. Increase in TREM2 gene dosage reduces steatohepatitis and sepsis-induced liver injury. (A) Experimental design used to evaluate the role of elevated TREM2 gene dosage in NAFLD progression and sepsis. (B and C) Body weight (B) and liver weight (C) of BAC-TREM2 and littermate WT control mice after a 10-week HFD. $n=11$ in WT group; $n=15$ in BAC-TREM2 group. Filled and open symbols refer to male and female, respectively. (D-F) Representative images of $\mathrm{H} \& \mathrm{E}$-stained and $\mathrm{ORO}$-stained liver sections (D). H\&E revealed tissue composition, macrovesicular fat, hepatocyte ballooning, and lobular inflammation. ORO visualized lipid droplets. Number of hepatocytes with macrovesicular fat or hepatocyte ballooning, lobular inflammation score, and percentage of ORO-positive area per HMF was determined by Imagej from 5 fields per section (E and F). $n=7$ in WT group; $n=11$ in BAC-TREM2 group. Scale bar: $100 \mu \mathrm{m}$. (G and $\mathbf{H}$ ) Levels of hepatic triglyceride (G) and ATP (H) from BAC-TREM2 and littermate WT mice after a 10-week HFD. $n=7$ in WT group; $n=11$ in BAC-TREM2 group. (I and J) Organ injury and bacteria burden at 24 hours after CLP. Male BAC-TREM2 and WT mice were fed with HFD for 10 weeks. After dietary intervention, mild polymicrobial sepsis was induced by CLP (24-gauge needle). At 24 hours after CLP, livers, lungs, and blood were collected for H\&E staining (I) and bacterial burden test (J), respectively. $n=6$ in WT group; $n=5$ in BAC-TREM2 group. Scale bar: $100 \mu$ m. (K) Hepatic ATP levels were quantified by luciferase assay. $n=6$ in WT group; $n=5$ in BAC-TREM2 group. Data are represented as the mean \pm SD. Significance was determined by an unpaired, 2-tailed Student's $t$ test. ${ }^{*} P<0.05$, ${ }^{* *} P<0.01,{ }^{* *} P<0.001$.

antigen or hepatitis $\mathrm{C}$ antibody, and treatment with medications that can cause hepatic steatosis. Based on the LA value $\leq 40 \mathrm{HU}$ criteria (hepatic steatosis area $>30 \%$, moderate-to-severe, refs. 16-19), 129 septic patients with NAFLD were identified. Clinical characteristics of 524 adults with sepsis according to hepatic steatosis categories are summarized in Supplemental Table 1. The study protocol was approved by the local institutional review board, and written informed consent was obtained from all subjects or their surrogates.
Liver tissues. Liver samples from NAFLD or apparently healthy patients were obtained from donors who underwent organ donation after cardiac death (DCD) with proper ethical approvals from institutional review boards. The exclusion criteria was the same as mentioned above. The NAFLD activity was evaluated by biopsies at cold ischemia in all graft livers. The grade of liver steatosis was determined according to international standards (46). Score 0 (non-NAFLD) indicates steatosis area < 5\%; score 1-3 (NAFLD) 


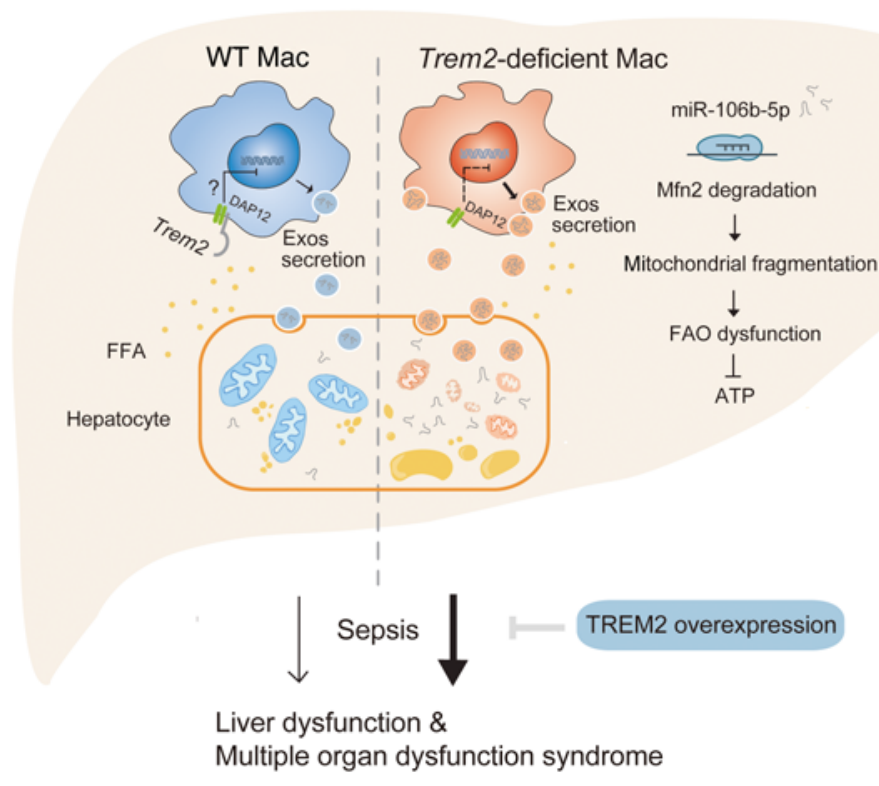

Figure 10. Schematic illustration of a mechanism through which macrophage TREM2 protects against NAFLD associated sepsis. Under a free fatty acidenriched (FFA-enriched) environment, macrophage TREM2 restrains the release of exosomes containing miR-106b-5p that targets to Mfn2 and leads to mitochondrial fragmentation in liver cells. The severely impaired energy supply in the Trem2-1- liver enhances susceptibility to sepsis. Strategies that target macrophage TREM2 may be an effective way to suppress steatohepatitis and relieve sepsis-induced liver dysfunction and multiple organ dysfunction syndrome. indicates steatosis area $\geq 5 \%$. All microscopic H\&E-stained sections and ORO-stained sections were evaluated by 2 pathologists in a double-blinded fashion. Nine non-NAFLD donors and 21 NAFLD donors were included. NAFLD activity and liver function of the 30 donors are summarized in Supplemental Table 2.

Mice. All animal experiments were reviewed and approved by the Animal Advisory Committee at Zhejiang University and Washington University in St. Louis for the care and use of laboratory animals. Trem2-deficient (Trem2-/-) mice on C57BL/6 background or BAC transgenic mice on C57BL/6 background and their littermate WT controls were generated as previously described $(12,41)$. All the mice were bred and maintained in a specific pathogen-free facility in standard cages at $22^{\circ} \mathrm{C}$ to $24^{\circ} \mathrm{C}$ with a 12 hour light-dark cycle. Eightweek-old mice were fed a normal control diet (NCD) (18.3\% protein, $10.2 \%$ fat, and $71.5 \%$ carbohydrates; Research Diets, D12450B) or a high fat diet (HFD) (18.1\% protein, $61.6 \%$ fat, and $20.3 \%$ carbohydrates; Research Diets, D12492) ad libitum for another 8 or 10 weeks. All fodder was replaced weekly to avoid contamination. For characterization of liver under HFD or NCD conditions in different genotype mice, all mice were fasted overnight prior to harvest.

RNA sequencing and differential expression analysis. Human/mouse liver samples or isolated mouse KCs from mice fed HFD for 8 weeks were subjected to parallel RNA sequencing using Illumina Hiseq platform (Novogene Experimental Department). Differential expression analysis was performed using the DESeq2 R package (1.16.1) (47). Read counts were used as input and normalized using built-in algorithms in DESeq2. Pairwise comparisons among the 2 conditions/groups were done on all genes. The resulting $P$ values were adjusted using the Benjamini and Hochberg's approach for controlling the false discovery rate. Genes with an adjusted $P$ value less than 0.05 found by DESeq 2 were assigned as differentially expressed. GO enrichment analysis of DEGs was implemented by the cluster Profiler R package, in which gene length bias was corrected (48). GO terms with corrected $P$ value less than 0.05 were considered significantly enriched by DEGs.

MicroRNA sequencing (miRNA-seq) was profiled by the small RNA sequencing analysis (Illumina). Using the KCs RNA-isolated from WT or Trem2 $2^{-/}$mice fed HFD for 8 weeks, a small RNA library was generated using the Illumina Truseq Small RNA Preparation kit following the manufacturer's guidelines. miRNA-seq reads were subjected to ACGT101-miR (LC Sciences) to sequence the data. Subsequently, unique sequences with length around 18-26 nucleotides were then mapped to all mouse miRNA hairpin sequences in miRBase 22.0 to identify known miRNAs and novel $3 p$ - and $5 p$-derived miRNAs (49). Differential expression of miRNAs based on normalized deepsequencing counts was analyzed by selectively using a Student's $t$ test. The significance threshold was set to 0.05 in each test. Sequencing data supporting these studies can be found at the Gene Expression Omnibus database under accession number GSE160022.

Kupffer cell depletion. Animals received 2 intravenous injections of sterile GdCl3 (20 mg/kg; Millipore Sigma, G7532) or vehicle control per week during the HFD feeding. After 8 weeks of dietary exposure, animals were fasted overnight before the tissues (blood, liver, adipose tissue) were harvested (32).

Cecal ligation and puncture model. Cecal ligation and puncture (CLP) was performed as described previously $(45,50)$. Briefly, mice were anesthetized with an i.p. injection of ketamine/xylazine (100 $\mathrm{mg} / \mathrm{kg}$ and $10 \mathrm{mg} / \mathrm{kg}$ ). After a $1.5 \mathrm{~cm}$ abdominal incision, the cecum was externalized, ligated (75\%) with 4:0 silk sutures, and punctured once with an 18-gauge needle (lethal) or 24-gauge needle (mild/less catastrophic) by one through-and-through puncture. We then internalized the cecum, closed the incision with 4:0 sutures, and resuscitated mice with $1 \mathrm{~mL}$ subcutaneous sterile saline. For survival experiments, mice were observed for mortality every 12 hours. In some experiments, mice were sacrificed at 24 hours post-CLP surgery, and then blood and organs were harvested.

Exosome purification and characterization. Exosomes were isolated by differential ultra-centrifugation as described previously $(6,33)$. BMDMs with different genotype were stimulated with $0.5 \mathrm{mM} \mathrm{PA}$ (Millipore Sigma, P0500) or control vehicle for 12 hours in exosome free medium. Dead cells and debris in culture media (depleted from serum-exosomes) were first removed by centrifuging at $350 \mathrm{~g}$ for 5 minutes, and then the supernatant underwent a second centrifugation 
at $2000 \mathrm{~g}$ for 20 minutes and a third centrifugation at 16,500 $g$ for 30 minutes. The supernatant was then subjected to ultracentrifugation at $110,000 \mathrm{~g}$ for 2 hours. After washing with sterile PBS and being recentrifuged at $110,000 \mathrm{~g}$ for 2 hours, the exosome-containing pellet was resuspended by PBS. Protein concentration was determined by bicinchoninic acid assay (Thermo Fisher Scientific, 23227). Further, exosome size distribution was measured by nanoparticle tracking analysis using the NanoSight LM10 (Malvern Panalytical). The device measures the Brownian motion of particles whose speed of motion, or diffusion coefficient, is related to particle size through the Stokes-Einstein equation.

Exosome in vivo treatment. Exosomes $(30 \mu \mathrm{g})$ derived from PA-treated ( $0.5 \mathrm{mM}, 12$ hours) BMDMs were rapidly injected into WT mice via tail vein with a volume of $200 \mu \mathrm{L}$. Exosomes were injected every week started at 7 weeks and mice were sacrificed at 10 weeks post-HFD feeding. To monitor the exosome trafficking after tail vein injection, exosomes were stained by lipophilic PKH26 fluorescent dye (Millipore Sigma, Mini26$1 \mathrm{KT}$ ) before injection. Liver and visceral adipose tissue (VAT) sections $(10 \mu \mathrm{m})$ were observed by Nikon A1R confocal microscope.

Primary hepatocyte isolation and culture. Primary hepatocytes were isolated from age-matched WT or Trem $2^{-/-}$male mice using released publications as reference (51). Briefly, mice were anesthetized by i.p. injection of ketamine/xylazine $(100 \mathrm{mg} / \mathrm{kg}$ and $10 \mathrm{mg} / \mathrm{kg})$. Livers were fully perfused through portal vein with liver perfusion buffer and then digested with dispase (Gibco, 17105-041) and collagenase type-I (Gibco, 17100-017) for 2 minutes at a rate of $2 \mathrm{~mL} / \mathrm{min}$. Then liver was excised, minced, and filtered through a $200 \mu \mathrm{m}$ mesh. Primary hepatocytes were washed by washing buffer containing $0.1 \mathrm{~g} / \mathrm{L}$ DNase (Sinopharm Chemical Reagent Co., Ltd, 64002860), $0.1 \mathrm{~g} / \mathrm{L}$ $\mathrm{MgSO}_{2} \cdot 7 \mathrm{H}_{2} \mathrm{O}$ and $0.1 \mathrm{~g} / \mathrm{L} \mathrm{MgCl}_{2} \cdot 6 \mathrm{H}_{2} \mathrm{O}$ and then separated via centrifugation at $110 \mathrm{~g}$ for 2 minutes. Primary hepatocytes were cultured in the DMEM supplemented with $10 \%$ FBS and $1 \%$ penicillin-streptomycin in a humidified incubator for 6 hours. The medium was then removed and replaced with fresh HepatoZYME-SFM medium (Gibco, 17705-021) supplemented with 1\% L-glutamine (Thermo Fisher Scientific, 25030081) and 1\% penicillin-streptomycin for future study.

Cell treatment. mmu-miR-106b-5p mimic or NC were synthesized by GenePharma (B02001 or B04003, respectively). In some experiments, miR-106b-5p was labeled with Cy3 before transfection. To transfect miRNAs into BMDMs, lipofectamine RNAiMAX reagent (Thermo Fisher Scientific, 13778150) was used to package miR-106b$5 \mathrm{p}$ mimic or NC. The transfection efficiencies were validated by qPCR.

For hepatocyte-macrophage coculture experiments, primary hepatocytes were isolated as above and seeded in the bottom chamber of transwell culture system with $0.4 \mathrm{~mm}$ polycarbonate filter $\left(1.5 \times 10^{5}\right.$ per well; Corning, 3413) and rested for 6 hours before use. Different genotype BMDMs or WT BMDMs transfected with mimic miR-106b-5p or NC were then seeded in the upper chamber $\left(1.5 \times 10^{5}\right.$ per well $)$ and cocultured with hepatocytes for 24 hours with $0.5 \mathrm{mM}$ PA in the culture medium.

For exosome treatment experiments, primary hepatocytes isolated from WT mice were seeded in a 12-well plate $\left(2.5 \times 10^{5}\right.$ per well $)$ and treated by $20 \mu \mathrm{g} / \mathrm{mL} \mathrm{WT}$ or Trem $2^{-/-}$BMDM-derived exosomes for 12 hours. To determine the Mfn2 expression of hepatocytes, BNL. CL2 cells were placed in a 12-well plate $\left(2.5 \times 10^{5}\right.$ cells per well $)$ and then transfected with miR-106b-5p mimic or NC for 24 hours.

TEM. For mitochondrial ultrastructure, liver tissue was dissected and fixed in $2.5 \%$ glutaraldehyde overnight at $4^{\circ} \mathrm{C}$, post-fixed in $1 \%$ osmium tetroxide for 80 minutes at $4^{\circ} \mathrm{C}$, dehydrated through a graded ethanol series, and embedded in epoxy resin. The tissue was sliced using an ultra-microtome (Leica EM UC7). Ultrathin sections (100 $\mathrm{nm}$ ) were mounted on copper grids and contrasted with $4 \%$ uranyl acetate and lead citrate. For exosome characters, exosomes resuspended in PBS as described above were pipetted onto $200 \mu \mathrm{m}$ mesh formvar/carbon grids (EMS) which had been glow-discharged for 15 seconds. Samples were incubated on grids for 30 seconds and subsequently negatively stained with a $2 \%$ uranyl acetate solution. Grids were observed with TEM (Tecnai G2 Spirit 120kV, Thermo Fisher Scientific) at the Center of Cyro-Electron Microscopy, Zhejiang University. The images for hepatocyte and exosomes were taken independently. Quantification of mitochondrial parameters in hepatocytes was performed using Image (length, diameter, and area; ref. 52). The percentage of damaged mitochondria was calculated. All quantifications were performed in double-bind fashion.

RNA isolation and detection of miRNA and mRNA. Total RNA was isolated either from tissue samples or cultured cells using TRIzol reagent (Thermo Fisher Scientific, 15596-026). The obtained total RNA was then reverse-transcribed into cDNA using the Reverse Transcription System (Promega, A3500) or the Mir-X miRNA firststrand synthesis kit (Takara, 638313) according to the manufacturer's instructions. TB Green premix Ex Taq II (Takara, RR820A) was used to quantify the PCR amplification products. The mRNA expression levels of the target genes were normalized to $\beta$-actin/GAPDH expression (Supplemental Table 3) and the miR-106b-5p expression levels were normalized to U6 (GeneCopoeia; mmu-miR-106b-5p primer: HmiRQP0029; U6 primer: HmiRQP9001). For exosome miRNA samples, 5 pg cel-miR-39 mimic (Qiagen, 219610) was added as an external control before isolated. The $2^{-\Delta \Delta C t}$ method was used to calculate relative gene expression levels.

Liver macrophage sorting and analysis. Liver macrophage sorting was performed using BD FACS Aria II flow cytometer (BD Biosciences). In brief, livers from HFD-fed mice were digested with collagenase type-I and DNase at $37^{\circ} \mathrm{C}$ after a low-pressure intraportal perfusion with EDTA and dispose solution. Cell suspension was filtered through Nylon gauze mesh, and centrifuged at $1000 \mathrm{~g}$ for 10 minutes. Then, single cells were isolated by using lymphocyte separation medium (DAKEWE, 7211011). After washed by PBS, liver nonparenchymal cells (NPCs) were stained by anti-mouse F4/80 (BM8, rat monoclonal, 1:50; Thermo Fisher Scientific, 12-4801-82) and anti-mouse CD11b (M1/70, rat monoclonal, 1:50; BD Biosciences, 553312) for 20 minutes while protected from light. Monocyte-derived macrophages ( $\mathrm{F} 4 / 80^{+} \mathrm{CD}$ $\left.11 \mathrm{~b}^{\text {hi }}\right)$ and $\mathrm{KCs}\left(\mathrm{F} 4 / 80^{\text {hi }} \mathrm{CD} 11 \mathrm{~b}^{+}\right)$were acquired on the sorting flow cytometer and analyzed using FlowJo version 10 software (Tree Star).

Statistics. All data are presented as mean \pm SD. Calculation for statistical significance was measured using 2-tailed Student's $t$ test or 1-way analysis of variance with Bonferroni corrections or Fisher's exact test as indicated in figure legends. Survival rates were analyzed using the log-rank test. Logistic regression analysis was used to examine covariate-adjusted associations between NAFLD and hospital mortality. Numbers of cohorts and $n$ values for each experiment are indicated in figure legends. All statistical analyses were performed using Prism 8.0 software (GraphPad Software Inc.), and $P$ values less than 0.05 were considered statistically significant.

Study approval. Human study protocols were approved by the institutional review board of Zhejiang University and written informed consent was obtained from all subjects or their surrogates. All animals were 
housed and procedures performed under a protocol approved either by the Animal Advisory Committee at Zhejiang University (protocol 2017-442) or Washington University in St. Louis (protocol 19-0981).

\section{Author contributions}

$\mathrm{XF}$ and MC designed and supervised the study. JH, XW, YJ, and BC designed the clinical study and collected the clinical data. JH, YZ, LS, and JX performed the transcriptomic profile and metabolites analyses. JH, YZ, JZ, PC, CL, HY, and KZ performed the in vivo experiments. PC, JZ, and SW performed the in vitro studies, as well as confocal and TEM microscopy. JH, PC, JZ, YJ, XF, and MC analyzed the data, generated the figures, and wrote the manuscript. QS and DW helped design the project and edited the manuscript. All authors discussed the results and approved the manuscript.

\section{Acknowledgments}

This work was supported by the National Key Research and Development Program of China (2018YFC2001904 to XF), the National
Natural Science Foundation of China (81720108025 and 81971865 to XF and JH), the Natural Science Foundation of Zhejiang Province (LR19H150001 to JH), and the Special Fund for Basic Scientific Research of Zhejiang University (2019QNA7039 to JH). We thank Menglong $\mathrm{Xu}$ for the technical support; Pinhao Li for the primary hepatocyte culture support; the technical assistant from the Center of Cryo-Electron Microscopy (CCEM); the Confocal Imaging Facility and the Histomorphology Facility at Zhejiang University School of Medicine; and the Molecular Imaging Center at Washington University in St. Louis for the technical support of ATP measurement.

Address correspondence to: Marco Colonna, Department of Pathology and Immunology, Washington University School of Medicine, 660 South Euclid, St. Louis, Missouri, 63110, USA. Phone: 314.362.0367; Email: mcolonna@wustl.edu. Or to: Xiangming Fang, Department of Anesthesiology, The First Affiliated Hospital, Zhejiang University School of Medicine, 79 Qingchun, Hangzhou, 310003, Zhejiang, China. Phone: 0086.571.88208006; Email: xmfang@zju.edu.cn.
1. Singer $\mathrm{M}$, et al. The third international consensus definitions for sepsis and septic shock (Sepsis-3). JAMA. 2016;315(8):801-810.

2. Iskander KN, et al. Sepsis: multiple abnormalities, heterogeneous responses, and evolving understanding. Physiol Rev. 2013;93(3):1247-1288.

3. Cecconi M, et al. Sepsis and septic shock. The Lancet. 2018;392(10141):75-87.

4. Musso G, et al. Non-alcoholic steatohepatitis: emerging molecular targets and therapeutic strategies. Nat Rev Drug Discov. 2016;15(4):249-274.

5. Leslie M. The liver's weighty problem. Science. 2015;349(6243):18-20.

6. Kim JY, et al. ER stress drives lipogenesis and steatohepatitis via caspase-2 activation of S1P. Cell. 2018;175(1):133-145.e15.

7. Garber K. The new liver epidemic. Nat Biotechnol. 2019;37(3):209-214.

8. Drew L. Fighting the fatty liver. Nature. 2017;550(7675):S102-S103.

9. Adams LA, et al. NAFLD as a risk factor for the development of diabetes and the metabolic syndrome: an eleven-year follow-up study. Am J Gastroenterol. 2009;104(4):861-867.

10. Houghton D, et al. The degree of hepatic steatosis associates with impaired cardiac and autonomic function. J Hepatol. 2019;70(6):1203-1213.

11. Choi HSJ, et al. Nonalcoholic steatohepatitis is associated with liver-related outcomes and allcause mortality in chronic hepatitis B. Hepatology. 2020;71(2):539-548.

12. Ulland TK, et al. TREM2 Maintains microglial metabolic fitness in Alzheimer's disease. Cell. 2017;170(4):649-663.e13.

13. Jaitin DA, et al. Lipid-associated macrophages control metabolic homeostasis in a trem2-dependent manner. Cell. 2019;178(3):686-698.e14.

14. Cochain C, et al. Single-cell RNA-seq reveals the transcriptional landscape and heterogeneity of aortic macrophages in murine atherosclerosis. Circ Res. 2018;122(12):1661-1674.

15. Chen QX, et al. Triggering receptor expressed on myeloid cells- 2 protects against polymicrobial sepsis by enhancing bacterial clearance. $\mathrm{Am} \mathrm{J}$ Respir Crit Care Med. 2013;188(2):201-212.
16. Kodama Y, et al. Comparison of CT methods for determining the fat content of the liver. AJR Am J Roentgenol. 2007;188(5):1307-1312.

17. Davidson LE, et al. Protocol for measurement of liver fat by computed tomography. J Appl Physiol (1985). 2006;100(3):864-868.

18. Boyce CJ, et al. Hepatic steatosis (fatty liver disease) in asymptomatic adults identified by unenhanced low-dose CT. AJR Am J Roentgenol. 2010;194(3):623-628

19. VanWagner LB, et al. Association of nonalcoholic fatty liver disease with subclinical myocardial remodeling and dysfunction: A population-based study. Hepatology. 2015;62(3):773-783.

20. Trevelin SC, et al. Diabetes mellitus and sepsis: a challenging association. Shock. 2017;47(3):276-287.

21. Irahara $\mathrm{T}$, et al. Alterations in energy substrate metabolism in mice with different degrees of sepsis. J Surg Res. 2018;227:44-51.

22. Liu TF, et al. NAD+-dependent sirtuin 1 and 6 proteins coordinate a switch from glucose to fatty acid oxidation during the acute inflammatory response. J Biol Chem. 2012;287(31):25758-25769.

23. Simcox J, et al. Global analysis of plasma lipids identifies liver-derived acylcarnitines as a fuel source for brown fat thermogenesis. Cell Metab. 2017;26(3):509-522.e6.

24. Mansouri A, et al. Mitochondrial dysfunction and signaling in chronic liver diseases. Gastroenterology. 2018;155(3):629-647.

25. Sunny NE, et al. Mitochondrial adaptation in nonalcoholic fatty liver disease: novel mechanisms and treatment strategies. Trends Endocrinol Metab. 2017;28(4):250-260.

26. Houten SM, et al. The biochemistry and physiology of mitochondrial fatty acid $\beta$-oxidation and its genetic disorders. Annu Rev Physiol. 2016;78:23-44.

27. Kazankov K, et al. The role of macrophages in nonalcoholic fatty liver disease and nonalcoholic steatohepatitis. Nat Rev Gastroenterol Hepatol. 2019;16(3):145-159.

28. Peterson KR, et al. Macrophage-targeted therapeutics for metabolic disease. Trends Pharmacol Sci. 2018;39(6):536-546.

29. Xiong XL, et al. Landscape of intercellular crosstalk in healthy and nash liver revealed by single-cell secretome gene analysis. Mol Cell. 2019;75(3):644-660.e5.

30. Wang YM, et al. TREM2 lipid sensing sustains the microglial response in an Alzheimer's disease model. Cell. 2015;160(6):1061-1071.

31. Grohmann M, et al. Obesity drives STAT-1dependent NASH and STAT-3-dependent HCC. Cell.2018;175(5):1289-1306.e20.

32. Huang W, et al. Depletion of liver Kupffer cells prevents the development of diet-induced hepatic steatosis and insulin resistance. Diabetes. 2010;59(2):347-357.

33. Ying W, et al. Adipose tissue macrophagederived exosomal miRNAs can modulate in vivo and in vitro insulin sensitivity. Cell. 2017;171(2):372-384.e12.

34. Awazawa M, et al. A microRNA screen reveals that elevated hepatic ectodysplasin A expression contributes to obesity-induced insulin resistance in skeletal muscle. Nat Med. 2017;23(12):1466-1473.

35. Jeppesen DK, et al. Reassessment of exosome composition. Cell. 2019;177(2):428-445.e18.

36. Varol D, et al. Dicer deficiency differentially impacts microglia of the developing and adult brain. Immunity. 2017;46(6):1030-1044.e8.

37. Zhang Y, et al. MicroRNA-106b induces mitochondrial dysfunction and insulin resistance in C2C12 myotubes by targeting mitofusin-2. Mol Cell Endocrinol. 2013;381(1-2):230-240.

38. Mishra P, et al. Proteolytic cleavage of Opa1 stimulates mitochondrial inner membrane fusion and couples fusion to oxidative phosphorylation. Cell Metab. 2014;19(4):630-641.

39. Hernandez-Alvarez MI, et al. Deficient endoplasmic reticulum-mitochondrial phosphatidylserine transfer causes liver disease. Cell. 2019;177(4):881-895.e17.

40. Kumar S, et al. Activation of mitofusin 2 by Smad2-RIN1 complex during mitochondrial fusion. Mol Cell. 2016;62(4):520-531.

41. Song WM, et al. Humanized TREM2 mice reveal microglia-intrinsic and -extrinsic effects of $\mathrm{R} 47 \mathrm{H}$ polymorphism. J Exp Med. 2018;215(3):745-760.

42. Lee CYD, et al. Elevated TREM2 gene dosage 
reprograms microglia responsivity and ameliorates pathological phenotypes in Alzheimer's disease models. Neuron. 2018;97(5):1032-1048.e5.

43. Strnad P, et al. Liver-guardian, modifier and target of sepsis. Nat Rev Gastroenterol Hepatol. 2017;14(1):55-66.

44. Bonnardel J, et al. Stellate cells, hepatocytes, and endothelial cells imprint the Kupffer cell identity on monocytes colonizing the liver macrophage niche. Immunity. 2019;51(4):638-654.e9.

45. Hou JC, et al. S1PR3 signaling drives bacterial killing and is required for survival in bacterial sepsis. Am J Respir Crit Care Med.2017;196(12):1559-1570.
46. Takahashi Y, Fukusato T. Histopathology of nonalcoholic fatty liver disease/nonalcoholic steatohepatitis. World J Gastroenterol. 2014;20(42):15539-15548.

47. Marschallinger J, et al. Lipid-droplet-accumulating microglia represent a dysfunctional and proinflammatory state in the aging brain. Nat Neurosci. 2020;23(2):194-208.

48. Young MD, et al. Gene ontology analysis for RNA-seq: accounting for selection bias. Genome Biol. 2010;11(2):R14.

49. Kozomara A, Griffiths-Jones S. miRBase: annotating high confidence microRNAs using deep sequencing data. Nucleic Acids Res. 2014;42(Database issue):D68-D73.

50. Patoli $\mathrm{D}$, et al. Inhibition of mitophagy drives macrophage activation and antibacterial defense during sepsis. JClin Invest. 2020;130(11):5858-5874.

51. Zhang T, et al. LXR $\alpha$ promotes hepatosteatosis in part through activation of MicroRNA-378 transcription and inhibition of Ppargc1 13 expression. Hepatology. 2019;69(4):1488-1503.

52. Fang EF, et al. Mitophagy inhibits amyloid- $\beta$ and tau pathology and reverses cognitive deficits in models of Alzheimer's disease. Nat Neurosci. 2019;22(3):401-412. 